Original paper

\title{
Patient-specific organ and effective dose estimates in pediatric oncology computed tomography
}

\author{
Yiming Gao ${ }^{\mathrm{a}, *}$, Brian Quinn ${ }^{\mathrm{a}}$, Neeta Pandit-Taskar ${ }^{\mathrm{b}}$, Gerald Behr ${ }^{\mathrm{b}}$, Usman Mahmood ${ }^{\mathrm{a}}$, \\ Daniel Long ${ }^{\mathrm{a}}$, X. George $\mathrm{Xu}^{\mathrm{c}}$, Jean St. Germain ${ }^{\mathrm{a}}$, Lawrence T. Dauer ${ }^{\mathrm{a}, \mathrm{b}}$ \\ a Department of Medical Physics, Memorial Sloan Kettering Cancer Center, 1275 York Avenue, New York, NY 10065, USA \\ ${ }^{\mathrm{b}}$ Department of Radiology, Memorial Sloan Kettering Cancer Center, 1275 York Avenue, New York, NY 10065, USA \\ ${ }^{\mathrm{c}}$ Department of Mechanical, Aerospace, and Nuclear Engineering, Rensselaer Polytechnic Institute, Troy, NY 12180, USA
}

\section{A R T I C L E I N F O}

\section{Keywords:}

Pediatric

Computed tomography

Diagnostic reference level

Organ dose

\begin{abstract}
A B S T R A C T
Purpose: Estimate organ and effective doses from computed tomography scans of pediatric oncologic patients using patient-specific information.

Materials and Methods: With IRB approval patient-specific scan parameters and patient size obtained from DICOM images and vendor-provided dose monitoring application were obtained for a cross-sectional study of 1250 pediatric patients from 0 through $20 \mathrm{y}$-olds who underwent head, chest, abdomen-pelvis, or chest-abdomen-pelvis CT scans. Patients were categorized by age. Organ doses and effective doses were estimated using VirtualDose $^{\mathrm{TM}}$ CT based on patient-specific information, tube current modulation (TCM), and age-specific realistic phantoms. CTDIvol, DLP, and dose results were compared with those reported in the literature. Results: CTDIvol and DLP varied widely as patient size varied. The 75th percentiles of CTDIvol and DLP were no greater than in the literature with the exception of head scans of 16-20y-olds and of abdomen-pelvis scans of larger patients. Eye lens dose from a head scan was up to $69 \mathrm{mGy}$. Mean organ doses agreed with other studies at maximal difference of $38 \%$ for chest and $41 \%$ for abdomen-pelvis scans. Mean effective dose was generally higher for older patients. The highest effective doses were estimated for the 16-20 y-olds as: head $3.3 \mathrm{mSv}$, chest $4.1 \mathrm{mSv}$, abdomen-pelvis $10.0 \mathrm{mSv}$, chest-abdomen-pelvis $14.0 \mathrm{mSv}$.

Conclusion: Patient-specific organ and effective doses have been estimated for pediatric oncologic patients from $<1$ through $20 \mathrm{y}$-olds. The effect of TCM was successfully accounted for in the estimates. Output parameters varied with patient size. CTDIvol and DLP results are useful for future protocol optimization.
\end{abstract}

\section{Introduction}

Computed Tomography (CT) is an important diagnostic imaging tool that helps save lives, but it does expose patients to ionizing radiation in addition to natural background radiation. On average, CT scans account for half of all medical radiation exposure in the United States (US) [1]. One in four persons in the US (per capita) had a CT scan in 2015 [2]. Concerns about the high scan number and the large contributions to medical exposure have led to campaigns and guidelines for justification and optimization of image quality and dose in the radiological community [3-6].

The advent of multi-detector CT (MDCT) has enabled rapid image acquisition without loss of spatial or contrast resolution. This is particularly useful in the pediatric oncologic setting, where cooperation with breath holds and motion avoidance are especially challenging for the young patients, many of whom have previously undergone numerous medical tests. Modern MDCT decreases reliance on and utilization of anesthesia [7]. However, MDCT technology itself does not directly decrease radiation exposure [8]. Recent successful decreases in pediatric exposure have resulted largely from national campaigns, which have raised awareness of the issue of CT parameter optimization within pediatric radiology practices [5]. This issue is of the utmost importance in the care of pediatric oncologic patients, as these patients tend to require numerous follow-up exams and subsequently receive relatively high cumulative dose.

In oncologic imaging, both pediatric and adult patients frequently undergo CT scans. While it is always important to justify and optimize CT radiation dose, it is particularly important to control the CT radiation dose to children. Compared to adults, pediatric patients are considered to have a higher risk of developing radiation-induced diseases

\footnotetext{
* Corresponding author.

E-mail addresses: gaoy1@mskcc.org (Y. Gao), quinnb@mskcc.org (B. Quinn), pandit-n@mskcc.org (N. Pandit-Taskar), behrg@mskcc.org (G. Behr), mahmoodu@mskcc.org (U. Mahmood), longd1@mskcc.org (D. Long), xug2@rpi.edu (X.G. Xu), stgermaj@mskcc.org (J. St. Germain), dauerl@mskcc.org (L.T. Dauer).
} 
Table 1

Patient demographics.

\begin{tabular}{|c|c|c|c|c|c|c|c|}
\hline \multirow[t]{2}{*}{ Age Group } & \multicolumn{5}{|c|}{ No. of Examinations } & \multirow{2}{*}{$\begin{array}{l}\text { Age (y) } \\
\text { Mean (Min-Max) }\end{array}$} & \multirow{2}{*}{$\begin{array}{l}\text { Weight (kg) } \\
\text { Mean (Min-Max) }\end{array}$} \\
\hline & Head & Chest & Abdomen and Pelvis & Chest, Abdomen, and Pelvis & Subtotal & & \\
\hline$<1$ year & 2 & 13 & 1 & 3 & 19 & $0.0(0.0-0.0)$ & $6.9(3.0-11.0)$ \\
\hline $1-5$ years & 41 & 55 & 17 & 98 & 211 & $2.8(1.0-4.0)$ & $14.4(7.0-30.0)$ \\
\hline $6-10$ years & 26 & 94 & 21 & 128 & 269 & $6.8(5.0-9.0)$ & $22.3(13.0-44.0)$ \\
\hline $11-15$ years & 20 & 130 & 26 & 79 & 255 & $12.3(10.0-14.0)$ & $49.0(23.0-142.0)$ \\
\hline $16-20$ years & 53 & 222 & 55 & 166 & 496 & $17.6(15.0-20.0)$ & $67.1(27.0-149.0)$ \\
\hline
\end{tabular}

Note: The mean and the minimum-to-maximum range in parenthesis are provided for age and body weight of patients in each age group.

Table 2

CT technique summary.

\begin{tabular}{|c|c|c|c|c|c|c|c|c|}
\hline Scanning region & $\begin{array}{l}\text { Tube potential } \\
(\mathrm{kVp})\end{array}$ & Tube current (mA) & $\begin{array}{l}\text { Revolution time } \\
\text { (s) }\end{array}$ & Collimation (mm) & Pitch & $\begin{array}{l}\text { CTDIvol } \\
\text { (mGy) }\end{array}$ & DLP (mGy·cm) & SSDE (mGy) \\
\hline Head & 100 or 120 & $\begin{array}{l}226.1 \\
(120.0-300.0)\end{array}$ & $1.0(1.0-1.0)$ & $17.3(10.0-40.0)$ & $1.01(0.98-1.07)$ & $\begin{array}{l}45.3 \\
(26-65.3)\end{array}$ & $\begin{array}{l}841.4 \\
(339.7-2036.3)\end{array}$ & $\mathrm{NA}^{*}$ \\
\hline Chest & 100 or 120 & $111.6(40.0-380.0)$ & $0.6(0.5-0.8)$ & $33.2(20.0-40.0)$ & $\begin{array}{l}1.26 \\
(0.98-1.38)\end{array}$ & $5.5(1.0-15.0)$ & $202.3(20.9-676.6)$ & $8.7(2.2-16.4)$ \\
\hline Abdomen and pelvis & 100 or 120 & $201.4(60.0-435.0)$ & $0.7(0.4-0.8)$ & $39.2(20.0-40.0)$ & $\begin{array}{l}1.22 \\
(0.98-1.38)\end{array}$ & $8.4(1.7-21.3)$ & $441.2(43.5-1218.9)$ & $\begin{array}{l}12.7 \\
(3.2-28.4)\end{array}$ \\
\hline $\begin{array}{l}\text { Chest, abdomen and } \\
\text { pelvis }\end{array}$ & 100 or 120 & $178.7(50.0-380.0)$ & $0.6(0.5-0.8)$ & $40.5(20.0-160.0)$ & $\begin{array}{l}1.31 \\
(0.98-1.38)\end{array}$ & $6.4(1.4-21.2)$ & $444.2(45.7-1750.1)$ & $\begin{array}{l}10.1 \\
(2.6-24.6)\end{array}$ \\
\hline
\end{tabular}

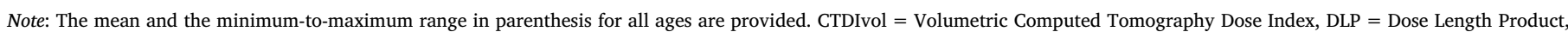
SSDE $=$ Size Specific Dose Estimate, NA = Not Applicable.

* SSDE for head scans were not used in this study.

Table 3

Mean and 75th percentile comparisons with international data for various pediatric age groups.

\begin{tabular}{|c|c|c|c|c|c|c|c|}
\hline \multirow[t]{2}{*}{ Exam } & \multirow[t]{2}{*}{ Age } & \multicolumn{3}{|l|}{ CTDIvol } & \multicolumn{3}{|l|}{ DLP } \\
\hline & & This study & Galanski et al. (2005) & Shrimpton et al. (2003) & This study & Galanski et al. (2005) & Shrimpton et al. (2003) \\
\hline \multirow[t]{5}{*}{ Head } & $<1 \mathrm{y}$ & $26(26)$ & $26(34)$ & $25(28)$ & $340(340)$ & $302(393)$ & $230(270)$ \\
\hline & $1-5 y$ & 34 (35) & $36(49)$ & $34(43)$ & $594(558)$ & $452(611)$ & $383(465)$ \\
\hline & $6-10 y$ & $36(38)$ & $44(58)$ & $44(51)$ & $599(607)$ & $582(711)$ & 508 (6 19) \\
\hline & $11-15 y$ & $49(48)$ & $53(65)$ & $56(64)$ & $810(815)$ & $764(920)$ & 694 (7 87) \\
\hline & $16-20 y$ & $58(60)$ & $61(60)$ & $56(64)$ & 1183 (1119) & $881(1100)$ & $694(787)$ \\
\hline \multirow[t]{5}{*}{ Chest } & $<1 \mathrm{y}$ & $1(1)$ & $3(4)$ & $11(12)$ & $28(29)$ & 40 (49) & 159 (204) \\
\hline & $1-5 y$ & $3(4)$ & $3(4)$ & $11(13)$ & $86(101)$ & $61(73)$ & $198(228)$ \\
\hline & $6-10 y$ & $4(5)$ & $5(6)$ & $14(17)$ & $124(153)$ & $102(128)$ & $303(368)$ \\
\hline & $11-15 y$ & $5(7)$ & $6(8)$ & $10(12)$ & $198(230)$ & $180(244)$ & $402(488)$ \\
\hline & $16-20 y$ & $7(8)$ & $11(10)$ & $10(12)$ & 277 (318) & $368(345)$ & $402(488)$ \\
\hline \multirow[t]{5}{*}{ Abdomen and pelvis } & $<1 \mathrm{y}$ & $2(2)$ & $3(4)$ & NA & $43(43)$ & $79(82)$ & NA \\
\hline & $1-5 y$ & $4(6)$ & $4(5)$ & NA & $162(220)$ & 115 (147) & NA \\
\hline & $6-10 y$ & $6(7)$ & $6(7)$ & NA & 247 (293) & $180(227)$ & NA \\
\hline & $11-15 y$ & $8(10)$ & $8(10)$ & $11(13)$ & $438(502)$ & 328 (402) & $473(534)$ \\
\hline & $16-20 y$ & 11 (12) & $13(15)$ & 11 (13) & 611 (719) & $568(980)$ & 473 (534) \\
\hline
\end{tabular}

Note: Dose data are mean (75th percentile). CTDIvol = Volumetric Computed Tomography Dose Index, DLP = Dose Length Product, NA $=$ Not Applicable

* Adult data is used for 16-20 years group.

${ }^{\dagger}$ Adult data is used for both 11-15 years group and 16-20 years group.

Table 4

Median and 75th percentile comparisons with U.S. data for 16-20 years group.

\begin{tabular}{|c|c|c|c|c|c|c|}
\hline \multirow[t]{2}{*}{ Examination } & \multicolumn{2}{|c|}{ CTDIvol (mGy) } & \multicolumn{2}{|c|}{ DLP (mGycm) } & \multicolumn{2}{|c|}{ SSDE (mGy) } \\
\hline & This study & Kanal et al. (2017) & This study & Kanal et al. (2017) & This study & Kanal et al. (2017) \\
\hline Head without contrast & $60(60)$ & $49(57)$ & 958 (1078) & $849(1011)$ & $58(60)$ & NA \\
\hline Chest without contrast & $6(8)$ & $9(12)$ & $259(338)$ & $334(443)$ & $9(11)$ & $11(15)$ \\
\hline Chest with contrast & $7(9)$ & $10(13)$ & 281 (359) & 353 (469) & $9(12)$ & $11(15)$ \\
\hline Abdomen and pelvis with contrast & $12(12)$ & $12(15)$ & $648(708)$ & $608(755)$ & 17 (19) & $15(18)$ \\
\hline Chest, abdomen, and pelvis with contrast & $9(11)$ & $12(15)$ & $637(878)$ & 779 (947) & $13(16)$ & $14(18)$ \\
\hline
\end{tabular}

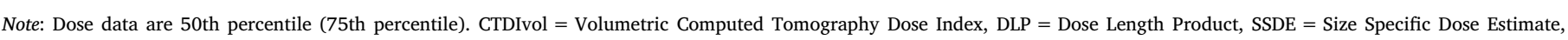
NA $=$ Not Applicable.

* Adult data is used for comparison. 
such as leukemia, thyroid, breast, brain, or skin cancer in their lifetime because of long life expectation as well as rapid cell replication and division in the body [9-12]. To accurately evaluate associated radiation-induced risks, knowledge of the doses to specific organs is recommended [12].

Volumetric CT dose index (CTDIvol), dose length product (DLP) and, more recently in vendor provided application DoseWatch ${ }^{\mathrm{TM}}$ (General Electric Inc., Milwaukee, USA), size-specific dose estimate (SSDE) are reported at the end of CT examinations [3,13], but typically without any organ dose information. Diagnostic reference level (DRL) is a tool recommended by the radiological protection community for the management and evaluation of CT dose quantities and for identification of unusually high doses [4,14]. DRLs are not limitations of diagnostic radiation dose or references of organ dose, but rather quantities for comparing protocols and for promoting protocol optimization and avoidance of unnecessary dose $[4,14]$. National or regional DRLs of CTDIvol, DLP and SSDE are published and available for institutional dose management [15-17].

Extra efforts are required to obtain specific organ dose by measurements or computational methods. One can utilize a humanoid phantom of tissue equivalent material and a number of detectors and perform CT scans on the phantom to obtain dose readings in locations designated as various organs [18-20]. On the other hand, one can also use sophisticated computational software to estimate organ dose for patients of various sizes and ages [21-23] or employ the use of sizebased computational methods and transverse patient size to estimate organ dose [24-26]. These methods have advantages and disadvantages and can be selected based on available resources and demands $[27,28]$.

Organ dose calculation for a large number of pediatric patients is complicated by the variations in patient age and patient body size and the various CT scan techniques, as well as the use of tube current modulation. Although studies have shown that individual patient-specific computational phantoms could be generated and Monte Carlo simulations performed to estimate organ dose [25,26], obtaining estimates in this manner currently takes a significant amount of time and is unrealistic for assessing doses in large numbers of patients. Moreover, the widely-used tube current modulation (TCM) technique changes the tube current angularly and/or longitudinally during CT scans to achieve an acceptable level of image quality with a lower average tube current, which redistributes the dose to organs and lowers the dose to some of them $[26,29,30]$. The complete TCM profile of a scan is usually proprietary and hard to obtain $[25,29]$, while a feasible way of obtaining longitudinal TCM is to extract the tube current of each image from the DICOM header [26]. In comparing several available organ dose estimation methods that generated patient-size based empirical functions by Monte Carlo simulations or phantom measurements [28], a reasonable and innovative approach would be to use modified computational software [21] that is capable of dose calculation for pediatric patients from $<1$ year (y) olds through $20 \mathrm{y}$-olds and can extract and directly apply the modulated tube current from DICOM images. The software was previously validated and compared to several other available tools as discussed in the literature $[21,28]$.

The aim of the present study was to estimate the organ and effective doses from computed tomography scans of oncologic pediatric patients with patient-specific information and to assess the reliability of these estimates by comparing them to estimates calculated with other methods from the literature [20,24-26].

\section{Methods}

\subsection{Patient population}

Institutional review board approval with a waiver of patient informed consent was obtained for this HIPAA-compliant, two-year retrospective study of CT scans of pediatric patients at a large academic cancer center performed during the period from September 2014 through September 2016. All DICOM images were anonymized prior to use. A total of 1250 cases were randomly selected to cover pediatric patients in five age categories: $<1 \mathrm{y}$-olds, $1-5 \mathrm{y}$-olds, 6-10y-olds, $11-15 y$-olds, and 16-20y-olds. In each age group, routine CT scans with or without imaging contrast of four body regions were selected: head (brain), chest, abdomen-pelvis (AP), and chest-abdomen-pelvis (CAP).

\subsection{CT examination techniques}

Records of pediatric CT scans were gathered by specifying time span via the vendor-provided dose monitoring application DoseWatch ${ }^{\mathrm{TM}}$ (General Electric Inc., Milwaukee, USA), and routine scans of the four aforementioned body regions were selected with in-house queries. Archived DICOM images of these CT scans were gathered through the HERMES GOLD ${ }^{\mathrm{Tm}}$ (Hermes Medical Solutions Inc., Stockholm, Sweden) system. All scans were performed on HD750 CT scanners (General Electric Inc., Milwaukee, USA). For organ dose and effective dose calculation, parameters were extracted from the DICOM headers of images; these included patient age (y), gender, body part examined, tube voltage $(\mathrm{kVp})$, tube current $(\mathrm{mA})$ of each image, revolution time (second), beam collimator width $(\mathrm{mm})$, pitch, bowtie filter type, CTDIvol (mGy), DLP (mGy*cm), and SSDE (mGy).

\subsection{Percentiles calculation for diagnostic reference level}

DRLs for specific CT protocols in the broad patient cohort were estimated at the 75th percentiles of the distributions of radiation dose quantities (e.g., CTDIvol, DLP and SSDE) [4,17]. The distributions of the CTDIvol, DLP and SSDE of the examinations were analyzed and the 75th percentiles were calculated and compared to DRLs from several national or regional surveys inside and outside the U.S. [15-17].

\subsection{Patient radiation dose assessment}

Patient organ dose was calculated using a modified version of the Monte Carlo-based CT dose calculation software VirtualDose ${ }^{\mathrm{TM}}$ CT (VDCT) (Virtual phantoms Inc., Albany, USA). The modification integrated VDCT with in-house computer programs to extract the tube current of each image and other parameters from DICOM headers. VDCT was also modified to enable automatic transfer of the parameters and automatic estimation of organ and effective doses.

Patients were matched to the available computational anthropomorphic phantoms in VDCT according to their age: $<1$ y-olds simulated by newborn phantoms, $1-5$ y-olds simulated by 1 y-old phantoms, 6-10y-olds simulated by $5 \mathrm{y}$-old phantoms, 11-15y-olds simulated by $10 \mathrm{y}$-old phantoms, and $16-20 \mathrm{y}$-olds simulated by $15 \mathrm{y}$ old phantoms. The body part examined was extracted and matched to the body region of the phantoms, with the start and end of scans defined as from top of head through the base of the skull for head scans, from the clavicles through the base of the lungs for chest scans, from the top of the liver through the pubic symphysis for AP scans, and from the clavicles through the pubic symphysis for CAP scans. Overscan of half of the beam width was assumed and added to each end of a simulated scan, since at least half of an extra rotation was needed for image reconstruction of helical CT scans [31]. Exam technique parameters were used to estimate organ dose as described previously [21], where tube current and time were applied in a slice-by-slice manner to address potential TCM technique. Results of organ doses and effective doses using tissue weighting factors from ICRP Publication No. 103 [32] were saved to a local database.

To account for the patient size variation within each age group, the ratio of patient-specific size-specific dose estimate (SSDE) to phantom SSDE was applied to all organ and effective dose results, as shown in the following equation: 
Table 5

Radiation dose to pediatric oncology patients by head CT scans.

\begin{tabular}{|c|c|c|c|c|c|c|c|c|c|c|}
\hline \multirow{2}{*}{$\begin{array}{l}\text { Patient age } \\
\text { Sex }\end{array}$} & \multicolumn{2}{|l|}{$<1 \mathrm{y}$} & \multicolumn{2}{|l|}{$1-5 y$} & \multicolumn{2}{|l|}{$6-10 y$} & \multicolumn{2}{|l|}{$11-15 y$} & \multicolumn{2}{|l|}{$16-20 y$} \\
\hline & $M(n=2)$ & $\mathrm{F}(\mathrm{n}=0)$ & $M(n=25)$ & $\mathrm{F}(\mathrm{n}=16)$ & $M(n=23)$ & $\mathrm{F}(\mathrm{n}=3)$ & $M(n=15)$ & $F(n=5)$ & $M(n=39)$ & $\mathrm{F}(\mathrm{n}=14)$ \\
\hline \multicolumn{11}{|l|}{ Effective Dose $(m S v)^{\dagger}$} \\
\hline Min & 1.9 & NA & 0.3 & 2.3 & 2.1 & 3.1 & 3.3 & 3.3 & 2.7 & 2.8 \\
\hline 25th percentile & 1.9 & NA & 2.3 & 2.4 & 3.1 & 3.1 & 3.4 & 3.3 & 3.3 & 3.4 \\
\hline Mean & 1.9 & NA & 2.3 & 2.6 & 3.1 & 3.2 & 3.5 & 3.4 & 3.3 & 3.3 \\
\hline 75th percentile & 1.9 & NA & 2.5 & 2.5 & 3.2 & 3.2 & 3.5 & 3.5 & 3.5 & 3.4 \\
\hline Max & 1.9 & NA & 3.0 & 4.5 & 3.2 & 3.2 & 4.4 & 3.5 & 3.5 & 3.4 \\
\hline Sex-averaged mean & 1.9 & & 2.4 & & 3.1 & & 3.5 & & 3.3 & \\
\hline By k-factor ${ }^{\dagger}$ & 3.7 & & 4.0 & & 2.4 & & 2.6 & & 2.5 & \\
\hline \multicolumn{11}{|l|}{ Organ Dose (mGy) } \\
\hline Red bone marrow & $\begin{array}{l}7.7 \\
(7.7,7.7)\end{array}$ & $\begin{array}{l}\text { NA } \\
\text { NA }\end{array}$ & $\begin{array}{l}9.0 \\
(1.1,11.4)\end{array}$ & $\begin{array}{l}10.2 \\
(9.3,17.2)\end{array}$ & $\begin{array}{l}11.2 \\
(7.4,11.7)\end{array}$ & $\begin{array}{l}11.6 \\
(11.5,11.8)\end{array}$ & $\begin{array}{l}15.5 \\
(14.3,20.3)\end{array}$ & $\begin{array}{l}14.9 \\
(14.4,15.2)\end{array}$ & $\begin{array}{l}15.3 \\
(12.5,17.2)\end{array}$ & $\begin{array}{l}15.6 \\
(12.9,16.1)\end{array}$ \\
\hline Breast & $\begin{array}{l}0.5 \\
(0.5,0.5)\end{array}$ & $\begin{array}{l}\text { NA } \\
\text { NA }\end{array}$ & $\begin{array}{l}0.5 \\
(0.1,0.6)\end{array}$ & $\begin{array}{l}0.6 \\
(0.5,1.0)\end{array}$ & $\begin{array}{l}0.5 \\
(0.3,0.6)\end{array}$ & $\begin{array}{l}0.6 \\
(0.6,0.6)\end{array}$ & $\begin{array}{l}0.3 \\
(0.3,0.4)\end{array}$ & $\begin{array}{l}0.3 \\
(0.3,0.3)\end{array}$ & $\begin{array}{l}0.3 \\
(0.3,0.3)\end{array}$ & $\begin{array}{l}0.1 \\
(0.1,0.1)\end{array}$ \\
\hline Colon & $\begin{array}{l}0.1 \\
(0.1,0.1)\end{array}$ & $\begin{array}{l}\text { NA } \\
\text { NA }\end{array}$ & $\begin{array}{l}0.1 \\
(0.0,0.1)\end{array}$ & $\begin{array}{l}0.1 \\
(0.1,0.1)\end{array}$ & $\begin{array}{l}0.0 \\
(0.0,0.0)\end{array}$ & $\begin{array}{l}0.0 \\
(0.0,0.0)\end{array}$ & $\begin{array}{l}0.0 \\
(0.0,0.0)\end{array}$ & $\begin{array}{l}0.0 \\
(0.0,0.0)\end{array}$ & $\begin{array}{l}0.0 \\
(0.0,0.0)\end{array}$ & $\begin{array}{l}0.0 \\
(0.0,0.0)\end{array}$ \\
\hline Lung & $\begin{array}{l}0.8 \\
(0.8,0.8)\end{array}$ & $\begin{array}{l}\text { NA } \\
\text { NA }\end{array}$ & $\begin{array}{l}0.8 \\
(0.1,1.1)\end{array}$ & $\begin{array}{l}1.0 \\
(0.9,1.7)\end{array}$ & $\begin{array}{l}1.3 \\
(0.8,1.4)\end{array}$ & $\begin{array}{l}1.4 \\
(1.4,1.4)\end{array}$ & $\begin{array}{l}0.7 \\
(0.7,0.9)\end{array}$ & $\begin{array}{l}0.7 \\
(0.7,0.8)\end{array}$ & $\begin{array}{l}0.5 \\
(0.4,0.5)\end{array}$ & $\begin{array}{l}0.6 \\
(0.5,0.6)\end{array}$ \\
\hline Stomach & $\begin{array}{l}0.2 \\
(0.2,0.2)\end{array}$ & $\begin{array}{l}\text { NA } \\
\text { NA }\end{array}$ & $\begin{array}{l}0.2 \\
(0.0,0.3)\end{array}$ & $\begin{array}{l}0.3 \\
(0.2,0.5)\end{array}$ & $\begin{array}{l}0.2 \\
(0.1,0.2)\end{array}$ & $\begin{array}{l}0.2 \\
(0.2,0.2)\end{array}$ & $\begin{array}{l}0.1 \\
(0.1,0.1)\end{array}$ & $\begin{array}{l}0.1 \\
(0.1,0.1)\end{array}$ & $\begin{array}{l}0.0 \\
(0.0,0.1)\end{array}$ & $\begin{array}{l}0.1 \\
(0.1,0.1)\end{array}$ \\
\hline Bladder & $\begin{array}{l}0.1 \\
(0.1,0.1)\end{array}$ & $\begin{array}{l}\text { NA } \\
\text { NA }\end{array}$ & $\begin{array}{l}0.0 \\
(0.0,0.0)\end{array}$ & $\begin{array}{l}0.0 \\
(0.0,0.1)\end{array}$ & $\begin{array}{l}0.0 \\
(0.0,0.0)\end{array}$ & $\begin{array}{l}0.0 \\
(0.0,0.0)\end{array}$ & $\begin{array}{l}0.0 \\
(0.0,0.0)\end{array}$ & $\begin{array}{l}0.0 \\
(0.0,0.0)\end{array}$ & $\begin{array}{l}0.0 \\
(0.0,0.0)\end{array}$ & $\begin{array}{l}0.0 \\
(0.0,0.0)\end{array}$ \\
\hline Esophagus & $\begin{array}{l}1.1 \\
(1.1,1.1)\end{array}$ & $\begin{array}{l}\text { NA } \\
\text { NA }\end{array}$ & $\begin{array}{l}2.0 \\
(0.2,2.7)\end{array}$ & $\begin{array}{l}2.3 \\
(2.1,4.1)\end{array}$ & $\begin{array}{l}2.9 \\
(1.2,3.0)\end{array}$ & $\begin{array}{l}3.1 \\
(3.0,3.1)\end{array}$ & $\begin{array}{l}1.5 \\
(1.4,1.9)\end{array}$ & $\begin{array}{l}1.5 \\
(1.4,1.5)\end{array}$ & $\begin{array}{l}0.8 \\
(0.7,0.9)\end{array}$ & $\begin{array}{l}0.8 \\
(0.7,0.8)\end{array}$ \\
\hline Gonads & $\begin{array}{l}0.0 \\
(0.0,0.0)\end{array}$ & $\begin{array}{l}\text { NA } \\
\text { NA }\end{array}$ & $\begin{array}{l}0.0 \\
(0.0,0.0)\end{array}$ & $\begin{array}{l}0.0 \\
(0.0,0.1)\end{array}$ & $\begin{array}{l}0.0 \\
(0.0,0.0)\end{array}$ & $\begin{array}{l}0.0 \\
(0.0,0.0)\end{array}$ & $\begin{array}{l}0.0 \\
(0.0,0.0)\end{array}$ & $\begin{array}{l}0.0 \\
(0.0,0.0)\end{array}$ & $\begin{array}{l}0.0 \\
(0.0,0.0)\end{array}$ & $\begin{array}{l}0.0 \\
(0.0,0.0)\end{array}$ \\
\hline Liver & $\begin{array}{l}0.3 \\
(0.3,0.3)\end{array}$ & $\begin{array}{l}\text { NA } \\
\text { NA }\end{array}$ & $\begin{array}{l}0.2 \\
(0.0,0.3)\end{array}$ & $\begin{array}{l}0.3 \\
(0.3,0.5)\end{array}$ & $\begin{array}{l}0.2 \\
(0.2,0.3)\end{array}$ & $\begin{array}{l}0.3 \\
(0.3,0.3)\end{array}$ & $\begin{array}{l}0.1 \\
(0.1,0.2)\end{array}$ & $\begin{array}{l}0.1 \\
(0.1,0.2)\end{array}$ & $\begin{array}{l}0.1 \\
(0.1,0.1)\end{array}$ & $\begin{array}{l}0.1 \\
(0.1,0.1)\end{array}$ \\
\hline Thyroid & $\begin{array}{l}3.2 \\
(3.2,3.2)\end{array}$ & $\begin{array}{l}\text { NA } \\
\text { NA }\end{array}$ & $\begin{array}{l}2.9 \\
(0.2,3.9)\end{array}$ & $\begin{array}{l}3.4 \\
(3.0,6.0)\end{array}$ & $\begin{array}{l}5.2 \\
(2.3,5.5)\end{array}$ & $\begin{array}{l}5.6 \\
(5.5,5.7)\end{array}$ & $\begin{array}{l}4.1 \\
(3.9,5.2)\end{array}$ & $\begin{array}{l}4.1 \\
(4.0,4.2)\end{array}$ & $\begin{array}{l}2.1 \\
(1.6,2.3)\end{array}$ & $\begin{array}{l}2.6 \\
(2.1,2.7)\end{array}$ \\
\hline Bone surface & $\begin{array}{l}10.3 \\
(10.3,10.3)\end{array}$ & $\begin{array}{l}\text { NA } \\
\text { NA }\end{array}$ & $\begin{array}{l}12.0 \\
(1.5,15.1)\end{array}$ & $\begin{array}{l}13.6 \\
(12.3,22.7)\end{array}$ & $\begin{array}{l}7.6 \\
(5.0,8.0)\end{array}$ & $\begin{array}{l}7.9 \\
(7.8,8.0)\end{array}$ & $\begin{array}{l}10.4 \\
(9.6,13.6)\end{array}$ & $\begin{array}{l}10.0 \\
(9.7,10.2)\end{array}$ & $\begin{array}{l}13.5 \\
(11.1,15.1)\end{array}$ & $\begin{array}{l}13.7 \\
(11.4,14.2)\end{array}$ \\
\hline Brain & $\begin{array}{l}29.8 \\
(29.8,29.8)\end{array}$ & $\begin{array}{l}\text { NA } \\
\text { NA }\end{array}$ & $\begin{array}{l}32.0 \\
(6.5,39.6)\end{array}$ & $\begin{array}{l}36.1 \\
(32.7,59.5)\end{array}$ & $\begin{array}{l}30.9 \\
(21.0,32.4)\end{array}$ & $\begin{array}{l}32.0 \\
(31.6,32.4)\end{array}$ & $\begin{array}{l}43.0 \\
(39.7,57.1)\end{array}$ & $\begin{array}{l}41.2 \\
(39.9,42.1)\end{array}$ & $\begin{array}{l}46.4 \\
(38.0,52.9)\end{array}$ & $\begin{array}{l}48.7 \\
(40.3,50.4)\end{array}$ \\
\hline Salivary Glands & $\begin{array}{l}5.2 \\
(5.2,5.2)\end{array}$ & $\begin{array}{l}\text { NA } \\
\text { NA }\end{array}$ & $\begin{array}{l}8.8 \\
(0.5,13.5)\end{array}$ & $\begin{array}{l}9.8 \\
(8.5,19.7)\end{array}$ & $\begin{array}{l}30.8 \\
(13.6,32.9)\end{array}$ & $\begin{array}{l}31.5 \\
(31.0,31.9)\end{array}$ & $\begin{array}{l}30.1 \\
(26.7,37.9)\end{array}$ & $\begin{array}{l}28.9 \\
(28.0,29.5)\end{array}$ & $\begin{array}{l}31.1 \\
(18.2,32.8)\end{array}$ & $\begin{array}{l}34.3 \\
(28.4,35.6)\end{array}$ \\
\hline Skin & $\begin{array}{l}5.8 \\
(5.8,5.8)\end{array}$ & $\begin{array}{l}\text { NA } \\
\text { NA }\end{array}$ & $\begin{array}{l}6.2 \\
(0.9,7.8)\end{array}$ & $\begin{array}{l}7.0 \\
(6.3,11.8)\end{array}$ & $\begin{array}{l}5.3 \\
(3.8,5.6)\end{array}$ & $\begin{array}{l}5.5 \\
(5.5,5.6)\end{array}$ & $\begin{array}{l}4.9 \\
(4.5,6.2)\end{array}$ & $\begin{array}{l}4.7 \\
(4.6,4.8)\end{array}$ & $\begin{array}{l}4.0 \\
(3.3,4.3)\end{array}$ & $\begin{array}{l}4.1 \\
(3.4,4.3)\end{array}$ \\
\hline Lens of eye & $\begin{array}{l}30.9 \\
(30.9,30.9)\end{array}$ & $\begin{array}{l}\text { NA } \\
\text { NA }\end{array}$ & $\begin{array}{l}35.1 \\
(1.1,38.7)\end{array}$ & $\begin{array}{l}39.6 \\
(36.5,57.7)\end{array}$ & $\begin{array}{l}40.1 \\
(28.8,42.1)\end{array}$ & $\begin{array}{l}41.7 \\
(41.1,42.2)\end{array}$ & $\begin{array}{l}54.9 \\
(51.0,69.0)\end{array}$ & $\begin{array}{l}52.9 \\
(51.3,54.1)\end{array}$ & $\begin{array}{l}66.4 \\
(54.5,72.3)\end{array}$ & $\begin{array}{l}68.6 \\
(56.8,71.0)\end{array}$ \\
\hline
\end{tabular}

Note: Organ dose is displayed as mean (minimum, maximum). $\mathrm{NA}=$ not applicable, $\mathrm{M}=$ male, $\mathrm{F}=$ female.

* Sex-specific examination number is given in parenthesis.

${ }^{\dagger}$ Mean effective doses were estimated using k-factors from AAPM report No. 96.

$D_{\text {patient }}=D_{\text {phantom }} \times S S D E_{\text {patient }} / S S D E_{\text {phantom }}$,

where $D_{\text {patient }}$ is the patient organ dose after SSDE correction, $D_{\text {phantom }}$ is the organ dose obtained from the VirtualDose software using the corresponding phantom, $S S D E_{\text {patient }}$ is the SSDE of the patient scan obtained from DoseWatch, and $S S D E_{\text {phantom }}$ is the SSDE of the corresponding phantom generated by multiplying the CT scan CTDIvol with the previously calculated f-factor (as described in AAPM report No. 220) for the scanned phantom region [33].

Effective dose was also roughly calculated using the DLP of the scans and the DLP-to-effective-dose conversion factors (known as " $k$ factors") [13]. For chest scans and AP scans, organ doses estimated in this study were compared to doses estimated with methods in the literature, which employed Monte Carlo method or measurements and generated patient-size based organ dose estimation equations [20,24-26]. Specifically, CTDIvol, SSDE and patient effective diameter of the scanned region were applied to these methods to estimate organ dose.

\subsection{Statistical analysis}

Statistical analyses were performed using the Matlab 2016b (Natick, Massachusetts, USA) software. Quantities calculated included minimum, 25th percentile, mean, 75th percentile, and maximum. Twoway ANOVA analyses (rows: different methods, columns: doses to different organs) were performed for comparisons between the results of this study and doses estimated with different methods in the literature. The null hypothesis was that the two compared sets of data were drawn from the same population with the same mean. The statistical significance level was defined as $\mathrm{p}<.05$.

\section{Results}

\subsection{Patient population}

Patient demographics are shown in Table 1. Of the 1250 CT scans 
Table 6

Radiation dose to pediatric oncology patients by chest CT scans.

\begin{tabular}{|c|c|c|c|c|c|c|c|c|c|c|}
\hline \multirow{2}{*}{$\begin{array}{l}\text { Patient age } \\
\text { Sex }\end{array}$} & \multicolumn{2}{|l|}{$<1 \mathrm{y}$} & \multicolumn{2}{|l|}{$1-5 y$} & \multicolumn{2}{|l|}{$6-10 y$} & \multicolumn{2}{|l|}{$11-15 y$} & \multicolumn{2}{|l|}{$16-20 y$} \\
\hline & $M(n=3)$ & $\mathrm{F}(\mathrm{n}=10)$ & $M(n=16)$ & $\mathrm{F}(\mathrm{n}=24)$ & $M(n=23)$ & $\mathrm{F}(\mathrm{n}=27)$ & $M(n=33)$ & $\mathrm{F}(\mathrm{n}=52)$ & $M(n=87)$ & $F(n=47)$ \\
\hline \multicolumn{11}{|l|}{ Effective Dose (mSv) } \\
\hline Min & 1.4 & 1.1 & 1.3 & 1.2 & 2.3 & 1.6 & 2.8 & 1.4 & 2.8 & 2.3 \\
\hline 25 th & 1.4 & 1.1 & 3.7 & 3.1 & 3.4 & 3.6 & 3.3 & 3.2 & 3.4 & 3.2 \\
\hline Mean & 1.4 & 1.3 & 3.6 & 3.4 & 4.1 & 3.9 & 3.7 & 4.0 & 4.2 & 3.9 \\
\hline 75 th & 1.5 & 1.4 & 4.1 & 4.0 & 5.0 & 4.1 & 4.1 & 4.7 & 4.9 & 4.7 \\
\hline $\operatorname{Max}$ & 1.5 & 1.6 & 5.5 & 5.3 & 6.2 & 6.0 & 5.4 & 5.9 & 7.5 & 6.5 \\
\hline Sex-averaged mean & 1.3 & & 3.5 & & 4.0 & & 3.9 & & 4.1 & \\
\hline By k-factor ${ }^{\dagger}$ & 1.1 & & 2.2 & & 2.2 & & 2.6 & & 3.9 & \\
\hline \multicolumn{11}{|l|}{ Organ Dose (mGy) } \\
\hline Red bone marrow & $\begin{array}{l}0.9 \\
(0.9,1.0)\end{array}$ & $\begin{array}{l}0.8 \\
(0.7,1.1)\end{array}$ & $\begin{array}{l}2.3 \\
(0.8,3.7)\end{array}$ & $\begin{array}{l}2.2 \\
(0.8,3.6)\end{array}$ & $\begin{array}{l}2.7 \\
(1.5,4.0)\end{array}$ & $\begin{array}{l}2.4 \\
(1.0,3.7)\end{array}$ & $\begin{array}{l}2.4 \\
(1.8,3.4)\end{array}$ & $\begin{array}{l}2.6 \\
(0.9,3.7)\end{array}$ & $\begin{array}{l}2.0 \\
(1.3,3.3)\end{array}$ & $\begin{array}{l}1.8 \\
(1.1,2.9)\end{array}$ \\
\hline Breast & $\begin{array}{l}2.9 \\
(2.8,3.0)\end{array}$ & $\begin{array}{l}2.6 \\
(2.2,3.2)\end{array}$ & $\begin{array}{l}6.2 \\
(2.2,9.3)\end{array}$ & $\begin{array}{l}6.0 \\
(2.2,9.6)\end{array}$ & $\begin{array}{l}7.7 \\
(4.2,12.2)\end{array}$ & $\begin{array}{l}7.6 \\
(3.1,11.8)\end{array}$ & $\begin{array}{l}6.9 \\
(4.9,10.5)\end{array}$ & $\begin{array}{l}7.5 \\
(2.9,11.7)\end{array}$ & $\begin{array}{l}7.7 \\
(5.1,15.0)\end{array}$ & $\begin{array}{l}7.6 \\
(4.2,12.8)\end{array}$ \\
\hline Colon & $\begin{array}{l}0.4 \\
(0.4,0.4)\end{array}$ & $\begin{array}{l}0.3 \\
(0.3,0.4)\end{array}$ & $\begin{array}{l}0.4 \\
(0.1,0.7)\end{array}$ & $\begin{array}{l}0.4 \\
(0.1,0.7)\end{array}$ & $\begin{array}{l}0.4 \\
(0.2,0.7)\end{array}$ & $\begin{array}{l}0.4 \\
(0.2,0.8)\end{array}$ & $\begin{array}{l}0.4 \\
(0.3,0.6)\end{array}$ & $\begin{array}{l}0.4 \\
(0.1,0.6)\end{array}$ & $\begin{array}{l}0.3 \\
(0.2,0.6)\end{array}$ & $\begin{array}{l}0.3 \\
(0.2,0.5)\end{array}$ \\
\hline Lung & $\begin{array}{l}2.9 \\
(2.8,3.1)\end{array}$ & $\begin{array}{l}2.6 \\
(2.2,3.3)\end{array}$ & $\begin{array}{l}7.9 \\
(2.8,11.8)\end{array}$ & $\begin{array}{l}7.5 \\
(2.7,11.7)\end{array}$ & $\begin{array}{l}8.9 \\
(4.8,13.5)\end{array}$ & $\begin{array}{l}8.3 \\
(3.4,12.4)\end{array}$ & $\begin{array}{l}8.8 \\
(6.6,12.4)\end{array}$ & $\begin{array}{l}9.5 \\
(3.5,14.0)\end{array}$ & $\begin{array}{l}9.3 \\
(6.2,16.4)\end{array}$ & $\begin{array}{l}8.8 \\
(5.3,14.5)\end{array}$ \\
\hline Stomach & $\begin{array}{l}1.3 \\
(1.3,1.4)\end{array}$ & $\begin{array}{l}1.2 \\
(1.0,1.5)\end{array}$ & $\begin{array}{l}3.5 \\
(1.2,5.4)\end{array}$ & $\begin{array}{l}3.3 \\
(1.2,5.3)\end{array}$ & $\begin{array}{l}5.1 \\
(2.9,8.0)\end{array}$ & $\begin{array}{l}4.8 \\
(2.0,9.1)\end{array}$ & $\begin{array}{l}3.9 \\
(2.6,5.9)\end{array}$ & $\begin{array}{l}4.0 \\
(1.5,6.2)\end{array}$ & $\begin{array}{l}4.3 \\
(2.7,7.6)\end{array}$ & $\begin{array}{l}4.9 \\
(2.7,8.2)\end{array}$ \\
\hline Bladder & $\begin{array}{l}0.1 \\
(0.1,0.1)\end{array}$ & $\begin{array}{l}0.1 \\
(0.0,0.1)\end{array}$ & $\begin{array}{l}0.1 \\
(0.0,0.2)\end{array}$ & $\begin{array}{l}0.1 \\
(0.0,0.2)\end{array}$ & $\begin{array}{l}0.1 \\
(0.0,0.1)\end{array}$ & $\begin{array}{l}0.1 \\
(0.0,0.1)\end{array}$ & $\begin{array}{l}0.0 \\
(0.0,0.0)\end{array}$ & $\begin{array}{l}0.0 \\
(0.0,0.0)\end{array}$ & $\begin{array}{l}0.0 \\
(0.0,0.1)\end{array}$ & $\begin{array}{l}0.0 \\
(0.0,0.0)\end{array}$ \\
\hline Esophagus & $\begin{array}{l}2.4 \\
(2.3,2.5)\end{array}$ & $\begin{array}{l}2.1 \\
(1.8,2.7)\end{array}$ & $\begin{array}{l}6.2 \\
(2.1,9.6)\end{array}$ & $\begin{array}{l}5.8 \\
(2.1,9.2)\end{array}$ & $\begin{array}{l}6.6 \\
(3.7,10.0)\end{array}$ & $\begin{array}{l}6.2 \\
(2.4,9.3)\end{array}$ & $\begin{array}{l}6.6 \\
(4.8,9.2)\end{array}$ & $\begin{array}{l}7.1 \\
(2.5,10.1)\end{array}$ & $\begin{array}{l}7.4 \\
(4.8,12.6)\end{array}$ & $\begin{array}{l}6.8 \\
(4.0,11.0)\end{array}$ \\
\hline Gonads & $\begin{array}{l}0.0 \\
(0.0,0.0)\end{array}$ & $\begin{array}{l}0.1 \\
(0.1,0.1)\end{array}$ & $\begin{array}{l}0.0 \\
(0.0,0.1)\end{array}$ & $\begin{array}{l}0.1 \\
(0.0,0.2)\end{array}$ & $\begin{array}{l}0.0 \\
(0.0,0.0)\end{array}$ & $\begin{array}{l}0.1 \\
(0.0,0.2)\end{array}$ & $\begin{array}{l}0.0 \\
(0.0,0.0)\end{array}$ & $\begin{array}{l}0.0 \\
(0.0,0.1)\end{array}$ & $\begin{array}{l}0.0 \\
(0.0,0.0)\end{array}$ & $\begin{array}{l}0.0 \\
(0.0,0.1)\end{array}$ \\
\hline Liver & $\begin{array}{l}2.1 \\
(2.0,2.2)\end{array}$ & $\begin{array}{l}1.8 \\
(1.6,2.3)\end{array}$ & $\begin{array}{l}4.3 \\
(1.5,6.5)\end{array}$ & $\begin{array}{l}4.0 \\
(1.4,6.4)\end{array}$ & $\begin{array}{l}6.1 \\
(3.4,9.3)\end{array}$ & $\begin{array}{l}5.7 \\
(2.3,10.7)\end{array}$ & $\begin{array}{l}5.7 \\
(3.9,8.7)\end{array}$ & $\begin{array}{l}5.9 \\
(2.2,9.0)\end{array}$ & $\begin{array}{l}6.1 \\
(3.8,10.8)\end{array}$ & $\begin{array}{l}6.2 \\
(3.4,10.4)\end{array}$ \\
\hline Thyroid & $\begin{array}{l}1.8 \\
(1.7,1.9)\end{array}$ & $\begin{array}{l}1.6 \\
(1.4,2.0)\end{array}$ & $\begin{array}{l}7.3 \\
(2.5,12.0)\end{array}$ & $\begin{array}{l}6.8 \\
(2.4,10.6)\end{array}$ & $\begin{array}{l}3.1 \\
(1.7,4.8)\end{array}$ & $\begin{array}{l}2.9 \\
(1.1,4.1)\end{array}$ & $\begin{array}{l}3.5 \\
(2.3,4.9)\end{array}$ & $\begin{array}{l}3.8 \\
(1.2,5.2)\end{array}$ & $\begin{array}{l}6.7 \\
(3.9,12.2)\end{array}$ & $\begin{array}{l}3.7 \\
(2.2,5.3)\end{array}$ \\
\hline Bone surface & $\begin{array}{l}0.9 \\
(0.9,1.0)\end{array}$ & $\begin{array}{l}0.8 \\
(0.7,1.0)\end{array}$ & $\begin{array}{l}2.2 \\
(0.8,3.6)\end{array}$ & $\begin{array}{l}2.1 \\
(0.8,3.4)\end{array}$ & $\begin{array}{l}2.7 \\
(1.5,4.1)\end{array}$ & $\begin{array}{l}2.5 \\
(1.0,3.8)\end{array}$ & $\begin{array}{l}2.5 \\
(1.8,3.4)\end{array}$ & $\begin{array}{l}2.7 \\
(0.9,3.7)\end{array}$ & $\begin{array}{l}3.1 \\
(2.0,5.2)\end{array}$ & $\begin{array}{l}2.9 \\
(1.7,4.6)\end{array}$ \\
\hline Brain & $\begin{array}{l}0.1 \\
(0.1,0.1)\end{array}$ & $\begin{array}{l}0.1 \\
(0.1,0.1)\end{array}$ & $\begin{array}{l}0.2 \\
(0.1,0.3)\end{array}$ & $\begin{array}{l}0.2 \\
(0.1,0.3)\end{array}$ & $\begin{array}{l}0.2 \\
(0.1,0.3)\end{array}$ & $\begin{array}{l}0.2 \\
(0.1,0.2)\end{array}$ & $\begin{array}{l}0.2 \\
(0.1,0.2)\end{array}$ & $\begin{array}{l}0.2 \\
(0.1,0.3)\end{array}$ & $\begin{array}{l}0.2 \\
(0.1,0.3)\end{array}$ & $\begin{array}{l}0.2 \\
(0.1,0.2)\end{array}$ \\
\hline Salivary Glands & $\begin{array}{l}0.4 \\
(0.4,0.4)\end{array}$ & $\begin{array}{l}0.4 \\
(0.3,0.5)\end{array}$ & $\begin{array}{l}1.2 \\
(0.4,1.9)\end{array}$ & $\begin{array}{l}1.0 \\
(0.4,1.6)\end{array}$ & $\begin{array}{l}0.8 \\
(0.4,1.2)\end{array}$ & $\begin{array}{l}0.7 \\
(0.3,1.0)\end{array}$ & $\begin{array}{l}0.7 \\
(0.5,1.0)\end{array}$ & $\begin{array}{l}0.8 \\
(0.2,1.1)\end{array}$ & $\begin{array}{l}0.7 \\
(0.5,1.3)\end{array}$ & $\begin{array}{l}0.6 \\
(0.4,0.9)\end{array}$ \\
\hline Skin & $\begin{array}{l}0.8 \\
(0.8,0.8)\end{array}$ & $\begin{array}{l}0.7 \\
(0.6,0.9)\end{array}$ & $\begin{array}{l}1.7 \\
(0.6,2.6)\end{array}$ & $\begin{array}{l}1.6 \\
(0.6,2.6)\end{array}$ & $\begin{array}{l}1.8 \\
(1.0,2.6)\end{array}$ & $\begin{array}{l}1.7 \\
(0.7,2.5)\end{array}$ & $\begin{array}{l}1.7 \\
(1.2,2.3)\end{array}$ & $\begin{array}{l}1.8 \\
(0.6,2.5)\end{array}$ & $\begin{array}{l}2.0 \\
(1.3,3.4)\end{array}$ & $\begin{array}{l}1.7 \\
(1.0,2.8)\end{array}$ \\
\hline Lens of eye & $\begin{array}{l}0.1 \\
(0.1,0.1)\end{array}$ & $\begin{array}{l}0.1 \\
(0.1,0.1)\end{array}$ & $\begin{array}{l}0.1 \\
(0.0,0.2)\end{array}$ & $\begin{array}{l}0.1 \\
(0.0,0.2)\end{array}$ & $\begin{array}{l}0.1 \\
(0.1,0.2)\end{array}$ & $\begin{array}{l}0.1 \\
(0.0,0.2)\end{array}$ & $\begin{array}{l}0.1 \\
(0.1,0.2)\end{array}$ & $\begin{array}{l}0.1 \\
(0.0,0.2)\end{array}$ & $\begin{array}{l}0.2 \\
(0.1,0.3)\end{array}$ & $\begin{array}{l}0.1 \\
(0.1,0.1)\end{array}$ \\
\hline
\end{tabular}

Note: Organ dose is displayed as mean (minimum, maximum). $\mathrm{NA}=$ not applicable, $\mathrm{M}=$ male, $\mathrm{F}=$ female.

* Sex-specific examination number is given in parenthesis.

${ }^{\dagger}$ Mean effective dose were estimated using k-factors from AAPM report No. 96.

evaluated in this study, 699 (59\%) were performed on male patients and $551(41 \%)$ on female patients (Table 1 ). For each of the age groups except the $<1$ y-olds, the number of scans was over 200 . The mean body weight of the patient age groups varied from $6.9 \mathrm{~kg}$ to $67.1 \mathrm{~kg}$. Pediatric patients with large body weight were found in the $11-15 y$ olds (maximum of $142 \mathrm{~kg}$ ) and the 16-20 y-olds (maximum of $149 \mathrm{~kg}$ ).

\subsection{CT examination techniques}

Table 2 lists mean values of CT technique parameters. The tube potential of the study cohort was $100 \mathrm{kVp}$ or $120 \mathrm{kVp}$. The tube current was modulated in $55 \%$ of the 1250 cases. Head scans had the highest effective mAs (mean, $224 \mathrm{mAs}$ ), followed by AP (116 mAs), CAP ( $87 \mathrm{mAs}$ ), and chest scans $(77 \mathrm{mAs})$. The variation (standard deviation or SD over mean) in the effective mAs was large, ranging from $27 \%$ for head scans to $42 \%$ for CAP scans. The bowtie filter type was medium filter for all head scans and body filter for all other scans.

\subsection{Percentiles calculation for diagnostic reference level}

The mean and 75th percentile of CTDIvol and DLP of various age groups from this study were compared to data from British and German national surveys (Table 3) $[15,16]$. The data from Shrimpton et al. [15] were for single-slice CT scanners, and their latest survey of multi-detector CT scanners [34] showed higher CTDIvol and DLP than their single-slice CT survey for all ages and protocols investigated. For CTDIvol the 75th percentiles in our study were no greater than those in the literature $[15,16]$ for any age- or region-specific groups except for AP scans of 1-5y-olds. For DLP, 75th percentiles for head scans of $16-20$ y-olds in our study were slightly higher $(2 \%)$ than those in the German survey. The 75th percentiles of DLP for chest scans of all age groups in our study were lower than the literature values. The 75th percentile DLP values of AP scans in our study were higher than those in the German survey for $1 \mathrm{y}$-olds through $15 \mathrm{y}$-olds, but the 75 th percentile CTDIvol values of these scans were very close to the literature 
Table 7

Radiation dose to pediatric oncology patients by Abdomen-Pelvis CT scans.

\begin{tabular}{|c|c|c|c|c|c|c|c|c|c|c|}
\hline \multirow{2}{*}{$\begin{array}{l}\text { Patient age } \\
\text { Sex }\end{array}$} & \multicolumn{2}{|l|}{$<1 \mathrm{y}$} & \multicolumn{2}{|l|}{$1-5 y$} & \multicolumn{2}{|l|}{$6-10 y$} & \multicolumn{2}{|l|}{$11-15 y$} & \multicolumn{2}{|l|}{$16-20 y$} \\
\hline & $\mathrm{M}(\mathrm{n}=0)$ & $\mathrm{F}(\mathrm{n}=1)$ & $M(n=11)$ & $F(n=6)$ & $\mathrm{M}(\mathrm{n}=3)$ & $\mathrm{F}(\mathrm{n}=17)$ & $\mathrm{M}(\mathrm{n}=8)$ & $\mathrm{F}(\mathrm{n}=18)$ & $M(n=24)$ & $\mathrm{F}(\mathrm{n}=13)$ \\
\hline \multicolumn{11}{|l|}{ Effective Dose (mSv) } \\
\hline Min & NA & 2.8 & 2.3 & 5.6 & 5.3 & 4.1 & 5.2 & 5.6 & 5.9 & 6.0 \\
\hline 25th & NA & 2.8 & 3.5 & 5.9 & 5.8 & 5.4 & 6.3 & 6.9 & 8.7 & 6.5 \\
\hline Mean & NA & 2.8 & 5.5 & 7.5 & 6.8 & 6.8 & 8.5 & 7.9 & 10.6 & 8.8 \\
\hline 75th & NA & 2.8 & 7.7 & 8.6 & 7.6 & 7.6 & 9.3 & 8.9 & 12.1 & 11.9 \\
\hline Max & NA & 2.8 & 8.4 & 10.3 & 7.7 & 10.5 & 17.9 & 11.9 & 15.4 & 14.8 \\
\hline Sex-averaged mean & 2.8 & & 6.2 & & 6.8 & & 8.1 & & 10.0 & \\
\hline By k-factor & 2.1 & & 4.9 & & 4.9 & & 6.6 & & 9.2 & \\
\hline \multicolumn{11}{|l|}{ Organ Dose (mGy) } \\
\hline Red bone marrow & $\begin{array}{l}\text { NA } \\
\text { NA }\end{array}$ & $\begin{array}{l}1.2 \\
(1.2,1.2)\end{array}$ & $\begin{array}{l}2.4 \\
(1.0,3.7)\end{array}$ & $\begin{array}{l}3.4 \\
(2.5,4.8)\end{array}$ & $\begin{array}{l}3.5 \\
(2.6,4.0)\end{array}$ & $\begin{array}{l}3.5 \\
(2.1,5.5)\end{array}$ & $\begin{array}{l}4.5 \\
(2.8,9.4)\end{array}$ & $\begin{array}{l}4.2 \\
(3.0,6.4)\end{array}$ & $\begin{array}{l}4.0 \\
(2.3,5.9)\end{array}$ & $\begin{array}{l}3.8 \\
(2.5,6.3)\end{array}$ \\
\hline Breast & $\begin{array}{l}\text { NA } \\
\text { NA }\end{array}$ & $\begin{array}{l}3.3 \\
(3.3,3.3)\end{array}$ & $\begin{array}{l}2.1 \\
(0.9,3.4)\end{array}$ & $\begin{array}{l}3.0 \\
(2.2,4.1)\end{array}$ & $\begin{array}{l}0.8 \\
(0.6,0.9)\end{array}$ & $\begin{array}{l}0.8 \\
(0.5,1.3)\end{array}$ & $\begin{array}{l}2.3 \\
(1.4,5.0)\end{array}$ & $\begin{array}{l}2.2 \\
(1.6,3.5)\end{array}$ & $\begin{array}{l}0.6 \\
(0.3,0.9)\end{array}$ & $\begin{array}{l}9.1 \\
(6.3,16.4)\end{array}$ \\
\hline Colon & $\begin{array}{l}\text { NA } \\
\text { NA }\end{array}$ & $\begin{array}{l}3.9 \\
(3.9,3.9)\end{array}$ & $\begin{array}{l}8.9 \\
(3.7,13.8)\end{array}$ & $\begin{array}{l}12.4 \\
(9.2,16.8)\end{array}$ & $\begin{array}{l}13.2 \\
(10.2,14.8)\end{array}$ & $\begin{array}{l}13.2 \\
(8.0,20.5)\end{array}$ & $\begin{array}{l}15.2 \\
(9.2,31.1)\end{array}$ & $\begin{array}{l}14.1 \\
(10.0,22.3)\end{array}$ & $\begin{array}{l}17.7 \\
(9.9,25.9)\end{array}$ & $\begin{array}{l}17.5 \\
(12.2,30.2)\end{array}$ \\
\hline Lung & $\begin{array}{l}\text { NA } \\
\text { NA }\end{array}$ & $\begin{array}{l}2.4 \\
(2.4,2.4)\end{array}$ & $\begin{array}{l}4.2 \\
(1.7,6.6)\end{array}$ & $\begin{array}{l}5.7 \\
(4.2,7.7)\end{array}$ & $\begin{array}{l}1.7 \\
(1.3,2.0)\end{array}$ & $\begin{array}{l}1.8 \\
(1.1,2.7)\end{array}$ & $\begin{array}{l}3.8 \\
(2.3,8.1)\end{array}$ & $\begin{array}{l}3.5 \\
(2.6,5.5)\end{array}$ & $\begin{array}{l}3.4 \\
(1.9,5.0)\end{array}$ & $\begin{array}{l}3.0 \\
(2.1,5.4)\end{array}$ \\
\hline Stomach & $\begin{array}{l}\text { NA } \\
\text { NA }\end{array}$ & $\begin{array}{l}3.8 \\
(3.8,3.8)\end{array}$ & $\begin{array}{l}8.3 \\
(3.4,13.1)\end{array}$ & $\begin{array}{l}11.5 \\
(8.5,15.5)\end{array}$ & $\begin{array}{l}10.9 \\
(8.3,12.5)\end{array}$ & $\begin{array}{l}11.0 \\
(6.8,16.9)\end{array}$ & $\begin{array}{l}13.9 \\
(8.1,29.1)\end{array}$ & $\begin{array}{l}12.7 \\
(9.3,19.7)\end{array}$ & $\begin{array}{l}15.8 \\
(8.8,23.0)\end{array}$ & $\begin{array}{l}14.6 \\
(10.2,26.3)\end{array}$ \\
\hline Bladder & $\begin{array}{l}\text { NA } \\
\text { NA }\end{array}$ & $\begin{array}{l}3.9 \\
(3.9,3.9)\end{array}$ & $\begin{array}{l}8.7 \\
(3.6,13.5)\end{array}$ & $\begin{array}{l}12.4 \\
(9.1,17.6)\end{array}$ & $\begin{array}{l}13.0 \\
(10.2,14.6)\end{array}$ & $\begin{array}{l}13.5 \\
(8.0,20.9)\end{array}$ & $\begin{array}{l}14.1 \\
(9.5,30.2)\end{array}$ & $\begin{array}{l}14.4 \\
(9.8,21.0)\end{array}$ & $\begin{array}{l}17.6 \\
(9.9,24.9)\end{array}$ & $\begin{array}{l}15.0 \\
(8.8,21.7)\end{array}$ \\
\hline Esophagus & $\begin{array}{l}\text { NA } \\
\text { NA }\end{array}$ & $\begin{array}{l}1.8 \\
(1.8,1.8)\end{array}$ & $\begin{array}{l}2.1 \\
(0.9,3.3)\end{array}$ & $\begin{array}{l}2.9 \\
(2.1,3.9)\end{array}$ & $\begin{array}{l}2.1 \\
(1.6,2.4)\end{array}$ & $\begin{array}{l}2.1 \\
(1.3,3.3)\end{array}$ & $\begin{array}{l}3.2 \\
(1.9,6.7)\end{array}$ & $\begin{array}{l}2.9 \\
(2.1,4.6)\end{array}$ & $\begin{array}{l}3.2 \\
(1.8,4.7)\end{array}$ & $\begin{array}{l}3.5 \\
(2.4,6.3)\end{array}$ \\
\hline Gonads & $\begin{array}{l}\text { NA } \\
\text { NA }\end{array}$ & $\begin{array}{l}3.5 \\
(3.5,3.5)\end{array}$ & $\begin{array}{l}8.5 \\
(3.6,13.2)\end{array}$ & $\begin{array}{l}12.1 \\
(9.0,17.0)\end{array}$ & $\begin{array}{l}13.3 \\
(10.5,14.9)\end{array}$ & $\begin{array}{l}12.2 \\
(7.2,19.1)\end{array}$ & $\begin{array}{l}15.7 \\
(10.6,33.1)\end{array}$ & $\begin{array}{l}12.8 \\
(8.7,19.2)\end{array}$ & $\begin{array}{l}21.4 \\
(11.8,29.9)\end{array}$ & $\begin{array}{l}14.5 \\
(8.5,21.7)\end{array}$ \\
\hline Liver & $\begin{array}{l}\text { NA } \\
\text { NA }\end{array}$ & $\begin{array}{l}3.8 \\
(3.8,3.8)\end{array}$ & $\begin{array}{l}8.6 \\
(3.5,13.5)\end{array}$ & $\begin{array}{l}11.8 \\
(8.7,15.9)\end{array}$ & $\begin{array}{l}10.6 \\
(8.1,12.1)\end{array}$ & $\begin{array}{l}10.6 \\
(6.7,16.4)\end{array}$ & $\begin{array}{l}13.3 \\
(7.8,27.9)\end{array}$ & $\begin{array}{l}12.1 \\
(8.9,18.8)\end{array}$ & $\begin{array}{l}16.1 \\
(8.9,23.4)\end{array}$ & $\begin{array}{l}14.1 \\
(9.9,25.4)\end{array}$ \\
\hline Thyroid & $\begin{array}{l}\text { NA } \\
\text { NA }\end{array}$ & $\begin{array}{l}0.3 \\
(0.3,0.3)\end{array}$ & $\begin{array}{l}0.6 \\
(0.2,0.9)\end{array}$ & $\begin{array}{l}0.8 \\
(0.6,1.1)\end{array}$ & $\begin{array}{l}0.3 \\
(0.2,0.3)\end{array}$ & $\begin{array}{l}0.3 \\
(0.2,0.4)\end{array}$ & $\begin{array}{l}0.3 \\
(0.2,0.6)\end{array}$ & $\begin{array}{l}0.3 \\
(0.2,0.4)\end{array}$ & $\begin{array}{l}0.2 \\
(0.1,0.4)\end{array}$ & $\begin{array}{l}0.3 \\
(0.2,0.5)\end{array}$ \\
\hline Bone surface & $\begin{array}{l}\text { NA } \\
\text { NA }\end{array}$ & $\begin{array}{l}1.2 \\
(1.2,1.2)\end{array}$ & $\begin{array}{l}2.3 \\
(0.9,3.5)\end{array}$ & $\begin{array}{l}3.2 \\
(2.3,4.5)\end{array}$ & $\begin{array}{l}4.0 \\
(3.0,4.6)\end{array}$ & $\begin{array}{l}4.0 \\
(2.4,6.4)\end{array}$ & $\begin{array}{l}5.0 \\
(3.1,10.4)\end{array}$ & $\begin{array}{l}4.7 \\
(3.3,7.2)\end{array}$ & $\begin{array}{l}6.1 \\
(3.4,8.8)\end{array}$ & $\begin{array}{l}5.7 \\
(3.7,9.3)\end{array}$ \\
\hline Brain & $\begin{array}{l}\text { NA } \\
\text { NA }\end{array}$ & $\begin{array}{l}0.1 \\
(0.1,0.1)\end{array}$ & $\begin{array}{l}0.1 \\
(0.0,0.1)\end{array}$ & $\begin{array}{l}0.1 \\
(0.1,0.1)\end{array}$ & $\begin{array}{l}0.0 \\
(0.0,0.0)\end{array}$ & $\begin{array}{l}0.0 \\
(0.0,0.1)\end{array}$ & $\begin{array}{l}0.0 \\
(0.0,0.1)\end{array}$ & $\begin{array}{l}0.0 \\
(0.0,0.1)\end{array}$ & $\begin{array}{l}0.0 \\
(0.0,0.0)\end{array}$ & $\begin{array}{l}0.0 \\
(0.0,0.0)\end{array}$ \\
\hline Salivary Glands & $\begin{array}{l}\text { NA } \\
\text { NA }\end{array}$ & $\begin{array}{l}0.2 \\
(0.2,0.2)\end{array}$ & $\begin{array}{l}0.2 \\
(0.1,0.3)\end{array}$ & $\begin{array}{l}0.3 \\
(0.2,0.4)\end{array}$ & $\begin{array}{l}0.1 \\
(0.1,0.1)\end{array}$ & $\begin{array}{l}0.1 \\
(0.1,0.2)\end{array}$ & $\begin{array}{l}0.1 \\
(0.1,0.2)\end{array}$ & $\begin{array}{l}0.1 \\
(0.1,0.2)\end{array}$ & $\begin{array}{l}0.1 \\
(0.0,0.1)\end{array}$ & $\begin{array}{l}0.1 \\
(0.0,0.1)\end{array}$ \\
\hline Skin & $\begin{array}{l}\text { NA } \\
\text { NA }\end{array}$ & $\begin{array}{l}1.8 \\
(1.8,1.8)\end{array}$ & $\begin{array}{l}3.6 \\
(1.5,5.5)\end{array}$ & $\begin{array}{l}5.0 \\
(3.7,6.8)\end{array}$ & $\begin{array}{l}4.1 \\
(3.2,4.6)\end{array}$ & $\begin{array}{l}4.1 \\
(2.5,6.4)\end{array}$ & $\begin{array}{l}4.8 \\
(3.0,10.0)\end{array}$ & $\begin{array}{l}4.5 \\
(3.2,6.7)\end{array}$ & $\begin{array}{l}5.5 \\
(3.1,8.0)\end{array}$ & $\begin{array}{l}4.8 \\
(3.1,7.8)\end{array}$ \\
\hline Lens of eye & $\begin{array}{l}\text { NA } \\
\text { NA }\end{array}$ & $\begin{array}{l}0.1 \\
(0.1,0.1)\end{array}$ & $\begin{array}{l}0.1 \\
(0.0,0.1)\end{array}$ & $\begin{array}{l}0.1 \\
(0.1,0.1)\end{array}$ & $\begin{array}{l}0.0 \\
(0.0,0.0)\end{array}$ & $\begin{array}{l}0.0 \\
(0.0,0.1)\end{array}$ & $\begin{array}{l}0.0 \\
(0.0,0.1)\end{array}$ & $\begin{array}{l}0.0 \\
(0.0,0.0)\end{array}$ & $\begin{array}{l}0.0 \\
(0.0,0.1)\end{array}$ & $\begin{array}{l}0.0 \\
(0.0,0.0)\end{array}$ \\
\hline
\end{tabular}

Note: Organ dose is displayed as mean (minimum, maximum). $\mathrm{NA}=$ not applicable, $\mathrm{M}=$ male, $\mathrm{F}=$ female.

* Sex-specific examination number is given in parenthesis.

${ }^{\dagger}$ Mean effective dose were estimated using k-factors from AAPM report No. 96.

values and lower than the 20 mGy DRL for 5 y-olds suggested by NCRP [14].

The median and 75th percentiles of CTDIvol, DLP and SSDE for 16-20 y-olds were compared with the data of adult CT scans from regional surveys inside the U.S. by Kanal et al. [17] (Table 4). For head scans both median and 75th percentiles were slightly higher $(22 \%$ and $7 \%$ respectively) than in the survey, but the 75th percentile of CTDIvol was still below the $75 \mathrm{mGy}$ DRL for adults suggested by NCRP [14]. For chest scans and CAP scans, the 50th and 75th percentiles were lower than in the survey. AP scans from our study had higher median DLP and higher 75th percentiles for SSDE than those in the U.S. survey. The sizespecific data of the survey confirmed the trend of increasing CTDIvol, DLP and SSDE with increasing patient body size for body scans [17].

\subsection{Patient radiation dose}

Tables 5-8 show the minimum, 25th percentiles, mean, 75th percentiles, and maximum of effective doses as well as the organ doses. In addition to the five age groups, the dose results are further broken down by gender. For 1007 of the 1250 scans that reported patient SSDE, organ dose was estimated and the SSDE ratio was applied to the dose results.

The mean effective dose estimated by VDCT showed an increasing trend as patients' ages increased, while effective dose roughly estimated with $\mathrm{k}$ factors showed similar trend except for head scans. The differences between VDCT estimated dose and $\mathrm{k}$ factors estimated dose were up to twofold for head scans, $70 \%$ for chest scans, $50 \%$ for AP scans, and $80 \%$ for CAP scans.

The five organs with the highest doses were: for head scans lens of eye, brain, salivary glands, red bone marrow and bone surface; for chest scans lung, breast, esophagus, liver, and thyroid; for AP scans gonads, colon, bladder, liver, and stomach; for CAP scans gonads, liver, colon, stomach, and bladder. 
Table 8

Radiation dose to pediatric oncology patients by Chest-Abdomen-Pelvis CT scans.

\begin{tabular}{|c|c|c|c|c|c|c|c|c|c|c|}
\hline \multirow{2}{*}{$\begin{array}{l}\text { Patient age } \\
\text { Sex }\end{array}$} & \multicolumn{2}{|l|}{$<1 \mathrm{y}$} & \multicolumn{2}{|l|}{$1-5 y$} & \multicolumn{2}{|l|}{$6-10 y$} & \multicolumn{2}{|l|}{$11-15 y$} & \multicolumn{2}{|l|}{$16-20 y$} \\
\hline & $M(n=3)$ & $\mathrm{F}(\mathrm{n}=0)$ & $M(n=58)$ & $\mathrm{F}(\mathrm{n}=39)$ & $M(n=73)$ & $\mathrm{F}(\mathrm{n}=54)$ & $M(n=47)$ & $\mathrm{F}(\mathrm{n}=30)$ & $M(n=88)$ & $\mathrm{F}(\mathrm{n}=63)$ \\
\hline \multicolumn{11}{|l|}{ Effective Dose (mSv) } \\
\hline Min & 2.7 & NA & 2.6 & 3.1 & 3.1 & 3.2 & 5.8 & 5.0 & 5.3 & 6.7 \\
\hline 25th & 2.9 & NA & 4.7 & 7.4 & 6.9 & 6.6 & 8.4 & 8.3 & 10.6 & 10.4 \\
\hline Mean & 4.3 & NA & 6.9 & 7.5 & 7.8 & 7.5 & 9.9 & 12.2 & 14.9 & 12.8 \\
\hline 75th & 5.9 & NA & 8.2 & 8.2 & 8.9 & 8.4 & 11.3 & 14.0 & 18.6 & 13.6 \\
\hline $\operatorname{Max}$ & 6.8 & NA & 10.3 & 9.1 & 12.9 & 12.3 & 15.5 & 24.6 & 25.7 & 23.7 \\
\hline Sex-averaged mean & 4.3 & & 7.2 & & 7.7 & & 10.8 & & 14.0 & \\
\hline By k-factor ${ }^{\dagger}$ & 3.5 & & 4.4 & & 4.3 & & 7.1 & & 11.4 & \\
\hline \multicolumn{11}{|l|}{ Organ Dose (mGy) } \\
\hline Red bone marrow & $\begin{array}{l}2.4 \\
(1.5,3.7)\end{array}$ & $\begin{array}{l}\text { NA } \\
\text { NA }\end{array}$ & $\begin{array}{l}3.7 \\
(1.4,5.9)\end{array}$ & $\begin{array}{l}4.0 \\
(1.6,4.8)\end{array}$ & $\begin{array}{l}4.5 \\
(1.7,7.5)\end{array}$ & $\begin{array}{l}4.3 \\
(1.8,7.2)\end{array}$ & $\begin{array}{l}5.8 \\
(3.2,8.8)\end{array}$ & $\begin{array}{l}7.1 \\
(3.1,13.6)\end{array}$ & $\begin{array}{l}6.4 \\
(2.4,10.7)\end{array}$ & $\begin{array}{l}6.1 \\
(3.1,10.9)\end{array}$ \\
\hline Breast & $\begin{array}{l}4.7 \\
(3.0,7.5)\end{array}$ & $\begin{array}{l}\text { NA } \\
\text { NA }\end{array}$ & $\begin{array}{l}6.4 \\
(2.4,11.7)\end{array}$ & $\begin{array}{l}7.1 \\
(2.9,8.6)\end{array}$ & $\begin{array}{l}8.0 \\
(3.2,13.6)\end{array}$ & $\begin{array}{l}7.8 \\
(3.0,13.3)\end{array}$ & $\begin{array}{l}8.4 \\
(5.2,13.9)\end{array}$ & $\begin{array}{l}11.2 \\
(4.6,24.0)\end{array}$ & $\begin{array}{l}11.9 \\
(5.7,20.9)\end{array}$ & $\begin{array}{l}11.9 \\
(5.7,25.9)\end{array}$ \\
\hline Colon & $\begin{array}{l}5.2 \\
(3.3,8.2)\end{array}$ & $\begin{array}{l}\text { NA } \\
\text { NA }\end{array}$ & $\begin{array}{l}7.9 \\
(3.0,11.3)\end{array}$ & $\begin{array}{l}8.8 \\
(3.6,10.6)\end{array}$ & $\begin{array}{l}9.3 \\
(3.9,16.4)\end{array}$ & $\begin{array}{l}9.0 \\
(3.9,15.5)\end{array}$ & $\begin{array}{l}12.4 \\
(7.2,22.5)\end{array}$ & $\begin{array}{l}15.8 \\
(6.0,32.8)\end{array}$ & $\begin{array}{l}18.3 \\
(5.7,33.5)\end{array}$ & $\begin{array}{l}18.8 \\
(8.3,40.0)\end{array}$ \\
\hline Lung & $\begin{array}{l}5.0 \\
(3.2,7.8)\end{array}$ & $\begin{array}{l}\text { NA } \\
\text { NA }\end{array}$ & $\begin{array}{l}8.6 \\
(3.2,15.0)\end{array}$ & $\begin{array}{l}9.3 \\
(3.8,11.2)\end{array}$ & $\begin{array}{l}9.2 \\
(3.5,14.8)\end{array}$ & $\begin{array}{l}8.9 \\
(3.7,14.3)\end{array}$ & $\begin{array}{l}11.3 \\
(6.9,15.5)\end{array}$ & $\begin{array}{l}13.4 \\
(6.2,24.1)\end{array}$ & $\begin{array}{l}15.2 \\
(6.2,24.6)\end{array}$ & $\begin{array}{l}13.0 \\
(6.8,23.1)\end{array}$ \\
\hline Stomach & $\begin{array}{l}5.1 \\
(3.3,8.1)\end{array}$ & $\begin{array}{l}\text { NA } \\
\text { NA }\end{array}$ & $\begin{array}{l}7.9 \\
(3.0,12.0)\end{array}$ & $\begin{array}{l}8.7 \\
(3.6,10.5)\end{array}$ & $\begin{array}{l}9.0 \\
(3.6,16.5)\end{array}$ & $\begin{array}{l}8.7 \\
(4.0,14.9)\end{array}$ & $\begin{array}{l}12.2 \\
(6.8,19.5)\end{array}$ & $\begin{array}{l}15.0 \\
(5.8,30.8)\end{array}$ & $\begin{array}{l}18.1 \\
(5.5,33.1)\end{array}$ & $\begin{array}{l}15.7 \\
(7.5,33.1)\end{array}$ \\
\hline Bladder & $\begin{array}{l}5.1 \\
(3.2,8.0)\end{array}$ & $\begin{array}{l}\text { NA } \\
\text { NA }\end{array}$ & $\begin{array}{l}7.8 \\
(3.0,12.4)\end{array}$ & $\begin{array}{l}8.6 \\
(3.5,10.4)\end{array}$ & $\begin{array}{l}9.3 \\
(3.8,15.2)\end{array}$ & $\begin{array}{l}9.6 \\
(4.1,17.0)\end{array}$ & $\begin{array}{l}12.1 \\
(6.7,22.8)\end{array}$ & $\begin{array}{l}16.7 \\
(6.1,35.7)\end{array}$ & $\begin{array}{l}17.9 \\
(6.2,32.0)\end{array}$ & $\begin{array}{l}17.6 \\
(7.5,32.5)\end{array}$ \\
\hline Esophagus & $\begin{array}{l}4.1 \\
(2.6,6.5)\end{array}$ & $\begin{array}{l}\text { NA } \\
\text { NA }\end{array}$ & $\begin{array}{l}6.5 \\
(2.5,12.4)\end{array}$ & $\begin{array}{l}7.1 \\
(2.9,8.5)\end{array}$ & $\begin{array}{l}6.9 \\
(2.6,11.6)\end{array}$ & $\begin{array}{l}6.7 \\
(2.9,10.9)\end{array}$ & $\begin{array}{l}8.6 \\
(5.0,12.0)\end{array}$ & $\begin{array}{l}10.0 \\
(4.9,17.4)\end{array}$ & $\begin{array}{l}12.3 \\
(5.0,19.4)\end{array}$ & $\begin{array}{l}10.5 \\
(5.4,18.3)\end{array}$ \\
\hline Gonads & $\begin{array}{l}3.2 \\
(2.1,5.1)\end{array}$ & $\begin{array}{l}\text { NA } \\
\text { NA }\end{array}$ & $\begin{array}{l}7.6 \\
(2.9,13.4)\end{array}$ & $\begin{array}{l}8.5 \\
(3.5,10.2)\end{array}$ & $\begin{array}{l}9.9 \\
(3.9,16.5)\end{array}$ & $\begin{array}{l}8.5 \\
(3.5,15.0)\end{array}$ & $\begin{array}{l}14.0 \\
(7.2,24.8)\end{array}$ & $\begin{array}{l}14.8 \\
(5.4,30.7)\end{array}$ & $\begin{array}{l}22.0 \\
(7.4,38.4)\end{array}$ & $\begin{array}{l}16.9 \\
(7.3,31.6)\end{array}$ \\
\hline Liver & $\begin{array}{l}5.3 \\
(3.4,8.3)\end{array}$ & $\begin{array}{l}\text { NA } \\
\text { NA }\end{array}$ & $\begin{array}{l}8.2 \\
(3.1,12.4)\end{array}$ & $\begin{array}{l}9.0 \\
(3.7,10.8)\end{array}$ & $\begin{array}{l}9.1 \\
(3.6,16.7)\end{array}$ & $\begin{array}{l}8.8 \\
(4.1,14.9)\end{array}$ & $\begin{array}{l}12.1 \\
(6.9,19.5)\end{array}$ & $\begin{array}{l}14.9 \\
(5.8,30.6)\end{array}$ & $\begin{array}{l}18.9 \\
(5.7,34.3)\end{array}$ & $\begin{array}{l}15.5 \\
(7.4,32.7)\end{array}$ \\
\hline Thyroid & $\begin{array}{l}2.9 \\
(1.9,4.6)\end{array}$ & $\begin{array}{l}\text { NA } \\
\text { NA }\end{array}$ & $\begin{array}{l}7.4 \\
(2.8,15.2)\end{array}$ & $\begin{array}{l}7.9 \\
(3.2,9.6)\end{array}$ & $\begin{array}{l}3.1 \\
(1.1,5.4)\end{array}$ & $\begin{array}{l}3.1 \\
(1.3,4.7)\end{array}$ & $\begin{array}{l}4.2 \\
(2.2,6.2)\end{array}$ & $\begin{array}{l}4.5 \\
(2.2,6.6)\end{array}$ & $\begin{array}{l}9.4 \\
(4.2,15.9)\end{array}$ & $\begin{array}{l}5.2 \\
(2.8,8.2)\end{array}$ \\
\hline Bone surface & $\begin{array}{l}2.2 \\
(1.4,3.5)\end{array}$ & $\begin{array}{l}\text { NA } \\
\text { NA }\end{array}$ & $\begin{array}{l}3.5 \\
(1.3,5.6)\end{array}$ & $\begin{array}{l}3.8 \\
(1.5,4.5)\end{array}$ & $\begin{array}{l}4.9 \\
(1.9,8.2)\end{array}$ & $\begin{array}{l}4.7 \\
(2.0,7.9)\end{array}$ & $\begin{array}{l}6.4 \\
(3.5,9.8)\end{array}$ & $\begin{array}{l}7.8 \\
(3.4,14.9)\end{array}$ & $\begin{array}{l}9.9 \\
(3.7,16.4)\end{array}$ & $\begin{array}{l}9.3 \\
(4.8,16.6)\end{array}$ \\
\hline Brain & $\begin{array}{l}0.2 \\
(0.1,0.3)\end{array}$ & $\begin{array}{l}\text { NA } \\
\text { NA }\end{array}$ & $\begin{array}{l}0.2 \\
(0.1,0.4)\end{array}$ & $\begin{array}{l}0.2 \\
(0.1,0.3)\end{array}$ & $\begin{array}{l}0.2 \\
(0.1,0.3)\end{array}$ & $\begin{array}{l}0.2 \\
(0.1,0.3)\end{array}$ & $\begin{array}{l}0.2 \\
(0.1,0.3)\end{array}$ & $\begin{array}{l}0.3 \\
(0.1,0.4)\end{array}$ & $\begin{array}{l}0.2 \\
(0.1,0.4)\end{array}$ & $\begin{array}{l}0.2 \\
(0.1,0.4)\end{array}$ \\
\hline Salivary Glands & $\begin{array}{l}0.7 \\
(0.5,1.1)\end{array}$ & $\begin{array}{l}\text { NA } \\
\text { NA }\end{array}$ & $\begin{array}{l}1.2 \\
(0.5,2.4)\end{array}$ & $\begin{array}{l}1.3 \\
(0.5,1.5)\end{array}$ & $\begin{array}{l}0.8 \\
(0.3,1.3)\end{array}$ & $\begin{array}{l}0.7 \\
(0.3,1.1)\end{array}$ & $\begin{array}{l}0.9 \\
(0.5,1.3)\end{array}$ & $\begin{array}{l}0.9 \\
(0.5,1.4)\end{array}$ & $\begin{array}{l}1.1 \\
(0.5,1.8)\end{array}$ & $\begin{array}{l}0.9 \\
(0.5,1.4)\end{array}$ \\
\hline Skin & $\begin{array}{l}2.9 \\
(1.9,4.6)\end{array}$ & $\begin{array}{l}\text { NA } \\
\text { NA }\end{array}$ & $\begin{array}{l}4.2 \\
(1.6,6.3)\end{array}$ & $\begin{array}{l}4.6 \\
(1.9,5.6)\end{array}$ & $\begin{array}{l}4.2 \\
(1.7,6.8)\end{array}$ & $\begin{array}{l}4.1 \\
(1.8,6.7)\end{array}$ & $\begin{array}{l}5.4 \\
(3.2,8.6)\end{array}$ & $\begin{array}{l}6.7 \\
(2.7,13.2)\end{array}$ & $\begin{array}{l}7.8 \\
(2.9,13.3)\end{array}$ & $\begin{array}{l}6.8 \\
(3.5,12.5)\end{array}$ \\
\hline Lens of eye & $\begin{array}{l}0.2 \\
(0.1,0.3)\end{array}$ & $\begin{array}{l}\text { NA } \\
\text { NA }\end{array}$ & $\begin{array}{l}0.1 \\
(0.1,0.3)\end{array}$ & $\begin{array}{l}0.2 \\
(0.1,0.2)\end{array}$ & $\begin{array}{l}0.1 \\
(0.0,0.2)\end{array}$ & $\begin{array}{l}0.1 \\
(0.0,0.2)\end{array}$ & $\begin{array}{l}0.1 \\
(0.1,0.2)\end{array}$ & $\begin{array}{l}0.2 \\
(0.1,0.2)\end{array}$ & $\begin{array}{l}0.3 \\
(0.1,0.4)\end{array}$ & $\begin{array}{l}0.1 \\
(0.1,0.2)\end{array}$ \\
\hline
\end{tabular}

Note: Organ dose is displayed as mean (minimum, maximum). $\mathrm{NA}=$ not applicable, $\mathrm{M}=$ male, $\mathrm{F}=$ female.

* Sex-specific examination number is given in parenthesis.

${ }^{\dagger}$ Mean effective dose were estimated using k-factors from AAPM report No. 96.

\section{Comparison to dose estimates by methods from the literature}

For chest scans the average doses to eight organs were compared between this study and those obtained with the methods of Moore et al. (M method), Tian et al. (Ti method), and Papadakis et al. (Pa method), as shown in Fig. 1 [20,25,26]. ANOVA analysis showed the doses estimated from this study and those estimated with the M method differed significantly $(\mathrm{p}<.05)$. Multiple comparison analysis showed the mean organ dose of this study was lower than that estimated with the M method [20], with the greatest difference being $-3.5 \mathrm{mGy}(-38 \%$, $5.8 \mathrm{mGy}$ versus $9.3 \mathrm{mGy}$ ) for $11-15 \mathrm{y}$-olds (where the negative sign indicates that doses in this study were lower). Estimated doses of the eight organs did not differ significantly between our method and $\mathrm{Ti}$ method $(p>.05)$ for any of the age groups compared. Dose estimates for five of the eight organs were compared between our method and Pa method [26], and significant differences in mean organ dose were found $(\mathrm{p}<.05)$ of $-1.1 \mathrm{mGy}(-11 \%, 8.7 \mathrm{mGy}$ versus $9.8 \mathrm{mGy}$, $16-20 \mathrm{y}$ old) and $0.8 \mathrm{mGy}(13 \%, 6.9 \mathrm{mGy}$ versus $6.1 \mathrm{mGy}, 1-5 \mathrm{y}$ old), where a negative sign again indicates doses of this study were lower.

For AP scans the average doses to seventeen organs were compared between our method and $\mathrm{M}$ method and that of Turner et al. (Tu method), as shown in Fig. 2 [20,24]. Mean organ dose differed significantly between our method and M method [20] ( $\mathrm{p}<.05)$, with differences ranging between $0.8 \mathrm{mGy}(10 \%, 8.5 \mathrm{mGy}$ versus $7.7 \mathrm{mGy}$, 1-5 y-olds) and $4.1 \mathrm{mGy}$ (41\%, $14.1 \mathrm{mGy}$ versus $10.0 \mathrm{mGy}, 16-20 \mathrm{y}-$ olds). No significant difference was found between our method and $\mathrm{Tu}$ method [24] ( $p>.05$ ) except for 16-20y-old, where the average difference was $-1.7 \mathrm{mGy}$ ( $-10 \%, 15.0 \mathrm{mGy}$ versus $16.7 \mathrm{mGy}$ ).

In summary the organ dose comparisons showed the dose estimation in our study was reasonably accurate: the maximal difference between VDCT doses and doses estimated by methods from the literature [20,24-26] was $38 \%$ for mean organ dose in chest scans and $41 \%$ for mean organ dose in AP scans. 

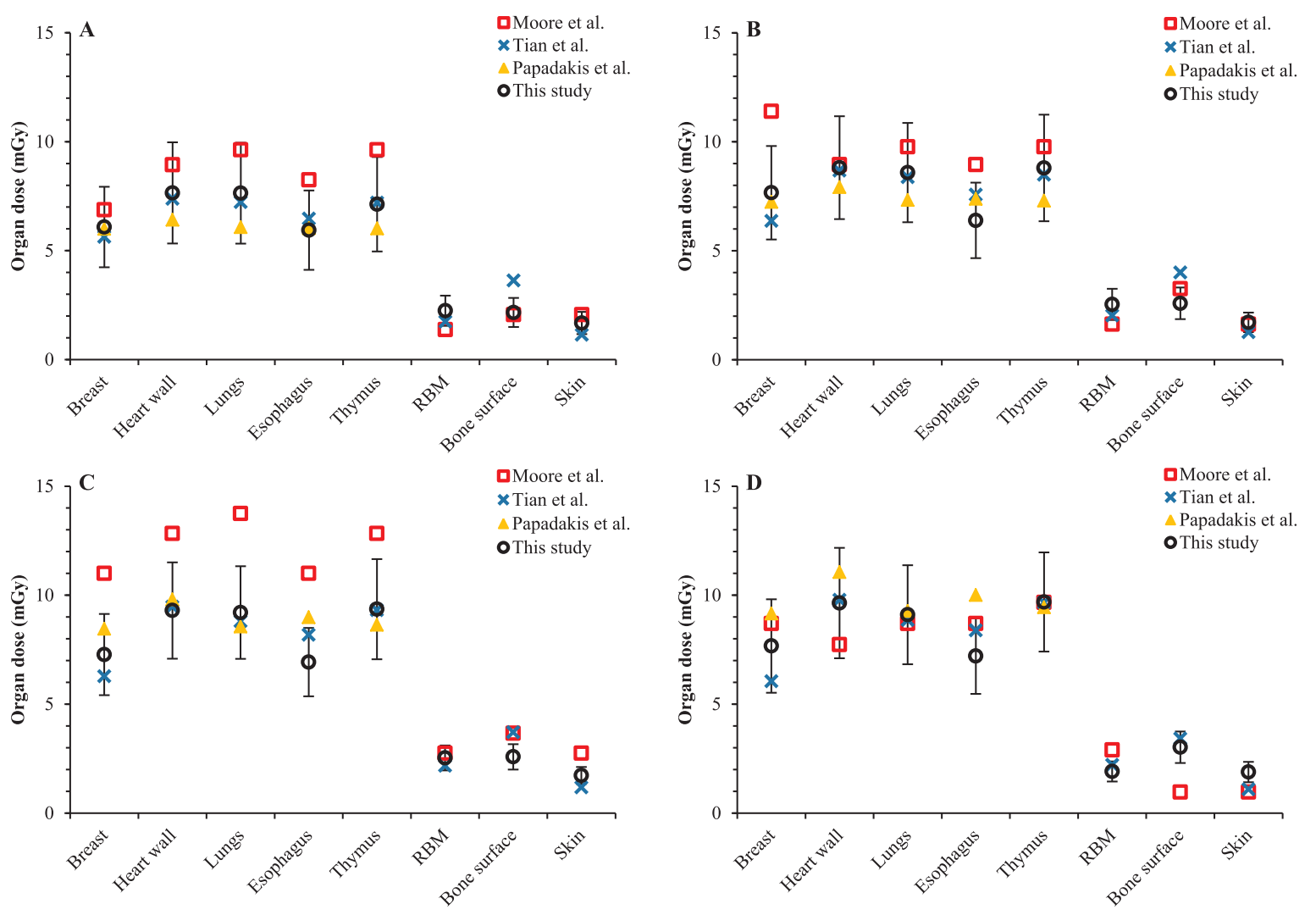

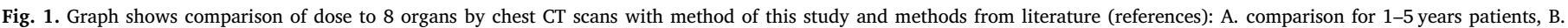

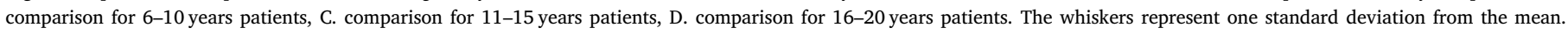
The $<1$ y-olds are not compared due to the limited number of examinations obtained in this study.

\section{Discussion}

To the best of our knowledge, this is the first study that estimates organ and effective dose for over a thousand pediatric patients receiving oncologic CT scans with TCM. The use of patient-specific scan parameters from CT images, individual patient size information from a vendor-provided dose monitoring application, and the sophisticated and validated Monte Carlo based dose estimator with realistic pediatric phantoms facilitated patient-specific organ and effective dose estimation. Regarding patient-specific organ dose estimation, our study did not only obtain the longitudinal TCM from DICOM headers of patient images as described in the literature, but also modified the computer code to automatically choose a phantom as close to a patient as possible based on age and sex, used the SSDE correction for size differences between the chosen phantom and the corresponding patient, and was able to process hundreds of CT scans in several minutes once all required information was obtained.

Except for the $<1$ y-olds, the number of exams was more than (or close to) 20 for each specific age group and body region, thus enabling the collection of representative organ dose results and the sufficient resultant data for valid statistical analyses.

The 11-15y-olds and 16-20 y-olds included patients of a very large size for their age (with body weight of $142 \mathrm{~kg}$ or more), for whom the use of an average-size human phantom was overly conservative. Therefore, for scans of the body regions, organ dose results were adjusted by the ratio of the SSDE of the patient to the SSDE of the phantom.

In our study, the CTDIvol and DLP were no greater than the DRLs from all literature surveys [15-17] except for head scans of 16-20 yolds, and the SSDE was lower than the DRL from the U.S. survey [17] except for AP scans with contrast. The calculation and analysis of the 75th percentiles of CTDIvol and DLP could be used to establish institutional DRLs to compare with reference levels and guide protocol optimization efforts that seek to reduce dose without compromising image quality [35]. The head scan dose percentiles suggest the opportunity for further optimization of protocols, and the AP scan dose 75th percentiles were affected by protocol adjustment for large patient size (up to $149 \mathrm{~kg}$ for $16-20 \mathrm{y}$-olds). A review of the data shows that for some of the 16-20y-olds, an adult head/brain protocol was utilized; this, coupled with some patients with extended scan length above the head, contributed to additional dose from head scans in this age group. Improvement on patient categorization by age and protocol selection is an ongoing mission at our institution.

Large variations in the scanner output were found to accommodate the large variations in patient sizes: For example, the variation in CTDIvol (one SD over mean) was $26 \%$ for head scans, $45 \%$ for chest scans, $46 \%$ for AP scans, and 56\% for CAP scans. In our study, the large variations in scan parameters accommodating variations in patient weight and size had led to variations in organ dose (one SD over mean) ranging from $13 \%$ through $50 \%$.

For head scans, comparison was not performed due to the lack of organ dose data from the literature that was based on anatomically realistic phantoms and could account for TCM. The doses to organs in the head were believed reasonably estimated because they were within $20 \%$ difference from CTDIvol. The dose to lens of eye reached as high as $69 \mathrm{mGy}$ for one brain CT scan for the 16-20 y-olds, and ten such scans during oncologic treatment fractions could lead to a total dose well above the 500-mGy dose threshold of the radiation-induced occurrence of cataract [36], suggesting that further optimization of lens of eye doses should perhaps be pursued [37].

For chest scans, doses estimated by the M method [20] were higher because their method could not account for the dose reduction effect of tube current modulation and their SSDE-to-dose factors (1.0-1.5 for inrange organs) led to estimated organ doses consistently greater than 

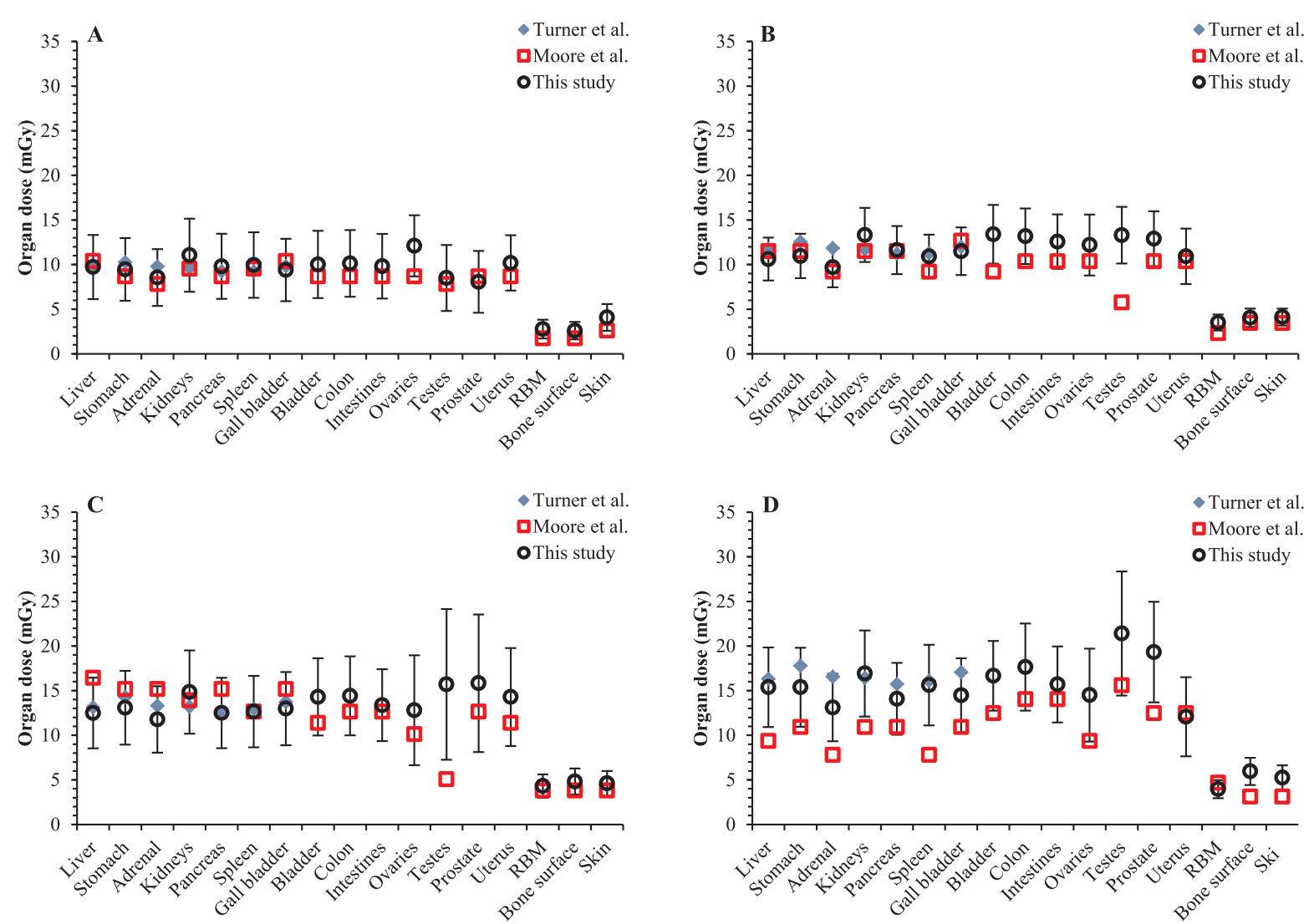

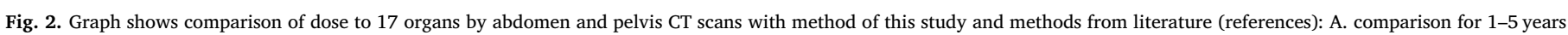

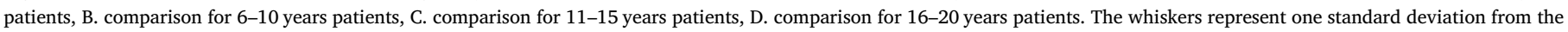
mean.

SSDE, whereas estimated organ doses in our study varied around SSDE [38]. For Ti method [25] and Pa method [26], extra steps were taken to obtain organ dose estimates. Tian et al. [25] and Papadakis et al. [26] generated their equations based on a 16-cm CTDI phantom, while in our study, the scan protocols in body regions were all based on a $32-\mathrm{cm}$ CTDI phantom regardless of patients' ages. Additional protocol-correction factors for Ti method [25] and Pa method [26] were generated in our comparisons, and these factors were the ratios of the CTDIvol of the specific scanner for $16-\mathrm{cm}$ phantom they used in their study to the CTDIvol of GE HD750 scanner for $32-\mathrm{cm}$ phantom in our study. Moreover, the water-equivalent diameter was required as input by $\mathrm{Pa}$ method [26] but unavailable in either DICOM headers or DoseWatch, and approximated by dividing the effective diameter with a factor of 1.22, which was the ratio of the two kinds of parameters of small patients for chest scans in the literature [33]. The extra efforts required by these methods may prevent clinical professionals from promptly and correctly estimating organ doses.

For AP scans, protocol-correction factor was not needed for $\mathrm{Tu}$ method [24], as their equations were based on a 32-cm CTDI phantom. However, Turner et al. only estimated doses to seven organs [24]. For 16-20 y-olds we used a pair of male and female $15 \mathrm{y}$ phantoms for dose estimation while Moore et al. used one adult female phantom [20]. The size and anatomical difference between $15 \mathrm{y}$ phantom and adult female phantom could have caused the large dose differences, and relatively small dose conversion factors were reported by Moore et al. using the adult phantom [20]. In addition the AP scan range of this study covered testes by considering overscan, while Moore et al. avoided direct irradiation of testes [20], and this range difference led to the large testes dose discrepancy as discussed in the literature [28].

Our study had several limitations. First, five pairs of pediatric phantoms were used to represent patients who were categorized into five age groups. Although patient size variation within each group was considered by applying the SSDE ratio of patient to phantom, ideally computational phantoms should be generated for each patient and Monte Carlo simulations performed on each one. However, this would require significant advances in computational speed. Second, only the longitudinal TCM was accounted for and the angular TCM was ignored because of lack of proprietary data. The angular TCM, especially the organ-based TCM could potentially decrease dose to sensitive organs such as lens of eye in head scans or female breasts in chest scans by up to $38 \%$, while at the same time increasing dose to other organs such as bone surface and bone marrow by about 10\% [39-42]. However, female breast dose reduction was lower (only up to 19\%) in clinical settings for patients than the reduction in experiments because of the lateral shift of breasts in supine position especially for elder patients [43]. Organ-based TCM was not used in any of the patient CT scans discussed in this study and based on literature we speculate using angular TCM the doses would be decreased by about $20 \%$ for superficial organs such as female breasts and the doses for bone surface and bone marrow would be increased by about 10\% [43-46]. Finally, our study provided very limited data on $<1 \mathrm{y}$-olds and could not provide definitive findings toward these patients.

\section{Conclusions}

Organ and effective doses were estimated for a large number of pediatric patients with CT scan-specific and patient-specific parameters, taking TCM into account. The 75th percentiles of scan parameters such as CTDIvol and DLP were calculated and found to be lower than DRLs in the literature except in head scans. These percentiles could be used to establish institutional DRLs and assist in protocol optimization in the future. As some adolescents in the study had large body sizes for their 
ages, SSDE ratios were used to account for patient size variations. Average organ dose estimates were found to be reasonably consistent (maximal difference of $38 \%$ for chest scans and $41 \%$ for AP scans) with estimates derived with other methods in the literature. Due to the variations in scan parameters, the relative standard deviation of organ dose was found to be as high as $50 \%$ of the mean dose. The large degree of variation in scan parameters called for patient-specific dose estimations, as performed in this study. Dose estimation methods utilizing CTDIvol with a multiplication factor should be utilized carefully to take into account the size of the CTDI phantom on which they are based. Dose to the lens of eye could be as high as $69 \mathrm{mGy}$ for one brain CT scan and several scans could lead to lens doses greater than the threshold of radiation-induced cataract $[36,47]$. As the method of dose estimation employed in this study allows consideration of the effects of TCM and accommodates a wide range of patients scanned with different parameters and is easy to use, it should be an appealing option until Monte Carlo simulations can be rapidly performed on computational phantoms generated for individual patients.

\section{Acknowledgements}

The authors would like to acknowledge the help of Dr. Hedvig Hricak, Mrs. Ada Muellner, and Dr. Robert Prins during the course of manuscript development. This research was funded in part through the NIH/NCI Cancer Center Support Grant P30 CA008748.Authors' contributions

YG collected and analyzed the data, drafted the manuscript, and participated in designing the study. BQ participated in designing the study and provided technical insights. NPT, GB, UM, DL, and JSG provided practical and clinical input and helped draft the manuscript. XGX provided the VirtualDose estimator and helped draft the manuscript. LTD conceived of and designed the study, provided technical oversight for dose calculation methods and results. All authors read and approved the manuscript.

\section{References}

[1] NCRP. Ionizing Radiation Exposure of the Population of the United States. NCRP Publications. Bethesda, MD2009.

[2] The Organisation for Economic Co-operation and Development (OECD). Computed tomography (CT) exams (indicator), 2016.

[3] AAPM. Size-Specific Dose Estimates (SSDE) in Pediatric and Adult Body CT Examinations. AAPM Report No. 204, AAPM Publications, Online 2011.

[4] ICRP. Radiation Protection in Medicine. ICRP Publication 105. 2007;37:1-63.

[5] Goske MJ, Applegate KE, Boylan J, Butler PF, Callahan MJ, Coley BD, et al. The Image Gently campaign: working together to change practice. AJR Am J Roentgenol 2008;190:273-4.

[6] Applegate KE, Frush DP. Image gently: a decade of international collaborations to promote appropriate imaging for children. J Am College Radiol: JACR 2017; 14:956-7.

[7] Pappas JN, Donnelly LF, Frush DP. Reduced frequency of sedation of young children with multisection helical CT. Radiology 2000;215:897-9.

[8] Valentin J. ICRP. Managing patient dose in multi-detector computed tomography (MDCT). ICRP Publication 102. Annals ICRP 2007;37:1-79.

[9] Brenner DJ, Hall EJ. Computed tomography-an increasing source of radiation exposure. N Engl J Med 2007;357:2277-84.

[10] Frush DP, Donnelly LF, Rosen NS. Computed tomography and radiation risks: what pediatric health care providers should know. Pediatrics 2003;112:951-7.

[11] Kalender WA. Dose in X-ray computed tomography. Phys Med Biol 2014;59:R129-50.

[12] UNSCEAR. Sources, effects and risks of ionizing radiation. UNSCEAR Publications. New York, NY: United Nations Scientific Committee on the Effects of Atomic Radiation; 2013.

[13] American Association of Physicists in Medicine (AAPM). The Measurement, Reporting, and Management of Radiation Dose in CT. AAPM Report No. 96. College Park, MD: AAPM, 2008.

[14] National Council on Radiation Protection and Measurements (NCRP). Reference Levels and Achievable Doses in Medical and Dental Imaging: Recommendations for the United States. Report No. 172. Bethesda, MD: NCRP, 2012.

[15] Shrimpton P, Hillier M, Lewis M, Dunn M. Doses from computed tomography (CT) examinations in the UK-2003 review. Chilton, UK: National Radiological Protection Board (NRPB); 2005. NRPB-W67 ed.

[16] Galanski M, Nagel H, Stamm G. Paediatric CT exposure practice in the Federal Republic of Germany. Results Nation-Wide Survey In 2005;6:2006.

[17] Kanal KM, Butler PF, Sengupta D, Bhargavan-Chatfield M, Coombs LP, Morin RL.
U.S. Diagnostic Reference Levels and Achievable Doses for 10 Adult CT Examinations. Radiology 2017:161911.

[18] Brisse HJ, Robilliard M, Savignoni A, Pierrat N, Gaboriaud G, De Rycke Y, et al Assessment of organ absorbed doses and estimation of effective doses from pediatric anthropomorphic phantom measurements for multi-detector row CT with and without automatic exposure control. Health Phys 2009;97:303-14.

[19] Zhang D, Li X, Gao Y, Xu XG, Liu B. A method to acquire CT organ dose map using OSL dosimeters and ATOM anthropomorphic phantoms. Med Phys 2013;40:081918.

[20] Moore BM, Brady SL, Mirro AE, Kaufman RA. Size-specific dose estimate (SSDE) provides a simple method to calculate organ dose for pediatric CT examinations. Med Phys 2014;41:071917.

[21] Ding A, Gao Y, Liu H, Caracappa PF, Long DJ, Bolch WE, et al. VirtualDose: a software for reporting organ doses from CT for adult and pediatric patients. Phys Med Biol 2015;60:5601-25.

[22] ImPACT. ImPACT CT dosimetry calculator, version 1.0.4. London: St. George's Healthcare NHS Trust; 2011.

[23] Stamm G, Nagel HD. CT-Expo V2.0.1: a tool for dose evaluation in computed tomography. Hannover 2014.

[24] Turner AC, Zhang D, Khatonabadi M, Zankl M, DeMarco JJ, Cagnon CH, et al. The feasibility of patient size-corrected, scanner-independent organ dose estimates for abdominal CT exams. Med Phys 2011;38:820-9.

[25] Tian X, Li X, Segars WP, Paulson EK, Frush DP, Samei E. Pediatric chest and abdominopelvic CT: organ dose estimation based on 42 patient models. Radiology 2014;270:535-47.

[26] Papadakis AE, Perisinakis K, Damilakis J. Development of a method to estimate organ doses for pediatric CT examinations. Med Phys 2016;43:2108.

[27] Brady Z, Cain TM, Johnston PN. Comparison of organ dosimetry methods and effective dose calculation methods for paediatric CT. Australas Phys Eng Sci Med 2012;35:117-34.

[28] Gao Y, Quinn B, Mahmood U, Long D, Erdi Y, St Germain J, et al. A comparison of pediatric and adult CT organ dose estimation methods. BMC Med Imaging 2017;17:28.

[29] Khatonabadi M, Kim HJ, Lu P, McMillan KL, Cagnon CH, DeMarco JJ, et al. The feasibility of a regional CTDIvol to estimate organ dose from tube current modulated CT exams. Med Phys 2013;40:051903.

[30] Schlattl H, Zankl M, Becker J, Hoeschen C. Dose conversion coefficients for paediatric CT examinations with automatic tube current modulation. Phys Med Biol 2012;57:6309-26.

[31] Schilham A, van der Molen AJ, Prokop M, de Jong HW. Overranging at multisection CT: an underestimated source of excess radiation exposure. Radiographics 2010;30:1057-67.

[32] ICRP. The 2007 Recommendations of the International Commission on Radiological Protection. ICRP Publication 103. 2007:37:1-332.

[33] American Association of Physicists in Medicine (AAPM). Use of Water Equivalent Diameter for Calculating Patient Size and Size-Specific Dose Estimates (SSDE) in CT. AAPM Report No. 220. College Park, MD: AAPM; 2014.

[34] Shrimpton P, Hillier M, Meeson S, Golding S. Doses from computed tomography (CT) examinations in the UK-2011 review. Chilton, UK: Public Health England; 2014. PHE-CRCE-013.

[35] Tonkopi E, Duffy S, Abdolell M, Manos D. Diagnostic reference levels and monitoring practice can help reduce patient dose from CT examinations. Am J Roentgenol 2017;208:1073-81.

[36] ICRP. ICRP Statement on Tissue Reactions and Early and Late Effects of Radiation in Normal Tissues and Organs - Threshold Doses for Tissue Reactions in a Radiation Protection Context. ICRP Publication 118. Annals of the ICRP. 2012;41.

[37] Vano E, Miller D, Dauer L. Implications in medical imaging of the new ICRP thresholds for tissue reactions. Ann ICRP 2015;44:118-28.

[38] Schlattl H, Zankl M, Becker J, Hoeschen C. Dose conversion coefficients for CT examinations of adults with automatic tube current modulation. Phys Med Biol 2010;55:6243-61.

[39] Duan X, Wang J, Christner JA, Leng S, Grant KL, McCollough CH. Dose reduction to anterior surfaces with organ-based tube-current modulation: evaluation of performance in a phantom study. AJR Am J Roentgenol 2011;197:689-95.

[40] Wang J, Duan X, Christner JA, Leng S, Yu L, McCollough CH. Radiation dose reduction to the breast in thoracic CT: comparison of bismuth shielding, organ-based tube current modulation, and use of a globally decreased tube current. Med Phys 2011;38:6084-92.

[41] Matsubara K, Sugai M, Toyoda A, Koshida H, Sakuta K, Takata T, et al. Assessment of an organ-based tube current modulation in thoracic computed tomography. J Appl Clin Med Phys. 2012;13:3731.

[42] Gandhi D, Crotty DJ, Stevens GM, Schmidt TG. Technical Note: phantom study to evaluate the dose and image quality effects of a computed tomography organ-based tube current modulation technique. Med Phys 2015;42:6572-8.

[43] Euler A, Szucs-Farkas Z, Falkowski AL, Kawel-Bohm N, D'Errico L, Kopp S, et al. Organ-based tube current modulation in a clinical context: dose reduction may be largely overestimated in breast tissue. Eur Radiol 2016;26:2656-62.

[44] Fu W, Tian X, Sturgeon GM, Agasthya G, Segars WP, Goodsitt MM, et al. CT breast dose reduction with the use of breast positioning and organ-based tube current modulation. Med Phys 2017;44:665-78.

[45] Fujii K, Nomura K, Muramatsu Y, Obara S, Akahane K, Kusumoto M. Organ Dose Evaluations Based on Monte Carlo Simulation for Ct Examinations Using Tube Current Modulation. Radiat Prot Dosim 2017;174:387-94.

[46] Kim JS, Kwon SM, Kim JM, Yoon SW. New organ-based tube current modulation method to reduce the radiation dose during computed tomography of the head: evaluation of image quality and radiation dose to the eyes in the phantom study. Radiol Med 2017;122:601-8.

[47] National Council on Radiation Protection and Measurements (NCRP). Guidance on Radiation Dose Limits for the Lens of the Eye. Commentary No. 26. Bethesda, MD: NCRP; 2016. 